\title{
The ISLAndS Project. II. The Lifetime Star Formation Histories of Six Andromeda dSphs
}

\author{
Evan D. Skillman ${ }^{1}$, Matteo Monelli ${ }^{2,3}$, Daniel R. Weisz ${ }^{4,18}$, Sebastian L. Hidalgo ${ }^{2,3}$, Antonio Aparicio ${ }^{2,3}$, Edouard J. Bernard ${ }^{5}$, \\ Michael Boylan-Kolchin ${ }^{6}$, Santi Cassisi ${ }^{7}$, Andrew A. Cole ${ }^{8}$, Andrew E. Dolphin ${ }^{9}$, Henry C. Ferguson ${ }^{10}$, Carme Gallart ${ }^{2,3}$, \\ Mike J. Irwin ${ }^{11}$, Nicolas F. Martin ${ }^{12,13}$, Clara E. Martínez-Vázquez ${ }^{2,3}$, Lucio Mayer ${ }^{14,15}$, Alan W. McConnachie ${ }^{16}$, \\ Kristen B. W. McQuinn ${ }^{6}$, Julio F. Navarro ${ }^{17}$, and Peter B. Stetson ${ }^{16}$ \\ ${ }^{1}$ Minnesota Institute for Astrophysics, University of Minnesota, Minneapolis, MN, USA; skillman@astro.umn.edu \\ ${ }^{2}$ Instituto de Astrofísica de Canarias (IAC). Vía Láctea s/n. E-38205-La Laguna, Tenerife, Canary Islands, Spain; monelli@iac.es, shidalgo@iac.es, aaj@iac.es, \\ carme@iac.es, clara.marvaz@gmail.com \\ ${ }^{3}$ Department of Astrophysics, University of La Laguna. Vía Láctea s/n. E-38206-La Laguna, Tenerife, Canary Islands, Spain \\ ${ }^{4}$ Astronomy Department, Box 351580, University of Washington, Seattle, WA, USA; dweisz@uw.edu \\ ${ }^{5}$ Laboratoire Lagrange (UMR7293), Observatoire de la Côte dAzur, F-06304 Nice, France; ebernard@oca.eu \\ ${ }^{6}$ Astronomy Department, University of Texas, Austin, TX, USA; mbk@astro.as.utexas.edu, kmcquinn@astro.as.utexas.edu \\ ${ }^{7}$ INAF-Osservatorio Astronomico di Collurania, Teramo, Italy; cassisi@oa-teramo.inaf.it \\ ${ }^{8}$ School of Physical Sciences, University of Tasmania, Hobart, Tasmania, Australia; andrew.cole@utas.edu.au \\ ${ }^{9}$ Raytheon; 1151 E. Hermans Road, Tucson, AZ 85706, USA; adolphin@ raytheon.com \\ ${ }^{10}$ Space Telescope Science Institute, 3700 San Martin Drive, Baltimore, MD 21218, USA; ferguson@ @stsci.edu \\ ${ }^{11}$ University of Cambridge, Madingley Road, Cambridge CB3 0HA, UK; mike@ast.cam.ac.uk \\ 12 Observatoire astronomique de Strasbourg, Universit de Strasbourg, CNRS, UMR 7550, 11 rue de l'Universit, F-67000 Strasbourg, France; nicolas.martin@astro. \\ unistra.fr \\ ${ }^{13}$ Max-Planck-Institut fr Astronomie, Knigstuhl 17, D-69117 Heidelberg, Germany \\ ${ }^{14}$ Institut für Theoretische Physik, University of Zurich, Zürich, Switzerland; lucio@ physik.unizh.ch \\ ${ }^{15}$ Department of Physics, Institut für Astronomie, ETH Zürich, Zürich, Switzerland; lucio@ @ phys.ethz.ch \\ ${ }^{16}$ Dominion Astrophysical Observatory, Herzberg Institute of Astrophysics, National Research Council, 5071 West Saanich Road, Victoria, British Columbia V9E \\ 2E7, Canada; peter.stetson@nrc-cnrc.gc.ca \\ ${ }^{17}$ Department of Physics and Astronomy, University of Victoria, BC V8P 5C2, Canada; jfn@uvic.ca \\ Received 2016 June 3; revised 2017 January 30; accepted 2017 February 13; published 2017 March 8
}

\begin{abstract}
The Initial Star formation and Lifetimes of Andromeda Satellites (ISLAndS) project employs Hubble Space Telescope imaging to study a representative sample of six Andromeda dSph satellite companion galaxies. Our main goal is to determine whether the star formation histories (SFHs) of the Andromeda dSph satellites demonstrate significant statistical differences from those of the Milky Way (MW). Our deep observations yield a time resolution at the oldest ages of $\sim 1 \mathrm{Gyr}$, allowing meaningful comparisons to the MW satellites. The six dSphs present a variety of SFHs (e.g., a significant range in quenching times, $\tau_{q}$, from 9 to $6 \mathrm{Gyr}$ ago) that are not strictly correlated with luminosity or present distance from M31. In agreement with observations of MW companions of similar mass, there is no evidence of complete quenching of star formation by the cosmic UV background responsible for reionization, but the possibility of a degree of quenching at reionization cannot be ruled out. We do not find significant differences between the SFHs of the members and non-members of the vast, thin plane of satellites. The SFHs of the ISLAndS M31 dSphs appear to be more uniform than those of the MW dSphs. Specifically, the primary difference between the SFHs of the ISLAndS dSphs and MW dSph companions of similar luminosities and host distances is the absence of late-quenching $\left(\tau_{q} \leqslant 5 \mathrm{Gyr}\right) \mathrm{dSphs}$ in the ISLAndS sample. Thus, models that can produce satellite populations with and without late-quenching satellites are of extreme interest.
\end{abstract}

Key words: galaxies: dwarf - galaxies: evolution - galaxies: photometry - galaxies: stellar content - galaxies: structure

\section{Introduction}

\subsection{Motivation: Testing for Bias in the MW Satellites}

The nearby dwarf galaxies of the Local Group are unique probes of galaxy formation and evolution over the entire history of the universe. Their proximity allows the study of their stellar, gaseous, and dark matter contents in unparalleled detail. However, they are not pristine, primeval systems, and their evolution is dependent upon both local and cosmic

* Based on observations made with the NASA/ESA Hubble Space Telescope, obtained at the Space Telescope Science Institute, which is operated by the Association of Universities for Research in Astronomy, Inc., under NASA contract NAS 5-26555. These observations are associated with programs \#13028, 13739.

18 Hubble Fellow. environmental factors. Thus, it is of tremendous importance to disentangle these effects for nearby galaxies and interpret them in a wider cosmological context. In currently favored hierarchical structure formation models, density fluctuations on the scale of dwarf galaxies collapse early and merge to form larger structures. However, the accretion of gas and its conversion to stars in dwarf galaxies is complicated and poorly understood, particularly at the earliest times. Cosmological simulations predict vastly more surviving dwarf galaxy sized halos than the number of observed dwarfs around the Milky Way (MW) and M31 ("the missing satellites problem," e.g., Kauffmann et al. 1993; Klypin et al. 1999; Moore 1999; Bullock 2010). The missing satellites problem cannot be solved by simply discovering more faint satellites to the MW as emphasized in the "too big to fail" problem (Boylan-Kolchin 
et al. 2011, 2012), which is repeated again for M31 (Collins et al. 2014; Tollerud et al. 2014). It seems clear that not all of these dark matter halos can retain baryons and form stars. Processes such as cosmic reionization (e.g., Efstathiou 1992; Bullock et al. 2000) and stellar feedback (e.g., Dekel \& Silk 1986; Tassis et al. 2003) are invoked to suppress star formation or remove the gas from some subset of dark matter halos.

Environmental effects are clearly important for the evolution of low-mass systems in the Local Group. Gas-poor, pressuresupported dwarf spheroidal galaxies (dSphs) are preferentially found as satellites of the MW and M31, whereas gas-rich, rotating dwarf irregulars (dIrrs) are preferentially found in isolated locales (e.g., van den Bergh 1994a; Grcevich \& Putman 2009). Additionally, the closest MW dSph companions (distances $\leqslant 100 \mathrm{kpc}$ ) have exclusively old stars with ages $\gtrsim$ $10 \mathrm{Gyr}$, while those more distant can show prominent young and intermediate-age stellar populations (e.g., van den Bergh 1994b; Mateo 1998; McConnachie 2012; Brown et al. 2014; Weisz et al. 2014a) and thus present a large variety of star formation histories (SFHs). It is not clear whether this configuration is a generic outcome of hierarchical structure formation models or a result of specific factors in our galaxy's history. Through detailed dynamical modeling, Mayer et al. (2001a, 2001b, 2006) have shown that "tidal stirring" can remove most of the gas from a dwarf galaxy and transform rotationally supported systems into pressure-supported systems. However, the existence of the isolated dSphs Cetus and Tucana, shown to be as old as the oldest MW companions (Monelli et al. 2010a, 2010b), point toward a multi-parameter process.

Very sophisticated models are being used to explore the environmental impacts on the evolution of dwarf galaxies (e.g., Ocvirk et al. 2014; Oñorbe et al. 2015; Wetzel et al. 2015a; Benítez-Llambay et al. 2016; Sawala et al. 2016; Wetzel et al. 2016). In addition, there is growing recognition that studying the nearest galaxies provides an observational window on highredshift galaxy evolution that even the next generation of highredshift galaxy surveys will not be able to provide (e.g., Weisz et al. 2014c; Boylan-Kolchin et al. 2015; Patej \& Loeb 2015; Boylan-Kolchin et al. 2016; Graus et al. 2016). Thus, it follows that obtaining observations of the nearest galaxies, which provide strong constraints on their lifetime SFHs, is critical to our progress.

Until very recently, the companions of the MW have been the only satellite dSphs with robust derivations of their SFHs at intermediate and old ages. The early SFHs of galaxies can only be revealed by observing resolved stars down to and below the oldest main-sequence turnoff (oMSTO; e.g., Gallart et al. 2005). Considering the unique role of the MW satellites as cosmological probes, it is vitally important that we understand whether their early SFHs are representative of satellite dSphs in the wider universe. The ISLAndS project is the first opportunity to test the representative nature of the early SFHs of the MW dSphs by obtaining complete, detailed SFHs for a representative sample of M31 satellites, the only other galaxy satellite system for which this is possible with the presently available technology. Our overall goal is to determine if the early evolution of the M31 companions is significantly different from the MW companions, and, if so, to determine the local or cosmic factors at play. Thus, we can address the question: are the dSph companions to the MW truly representative of dSph galaxies in general?

\subsection{M31 versus the MW}

Is there any reason to suspect that the satellite populations of M31 and the MW could be significantly different? There are significant differences between the properties of M31 and the MW (van den Bergh 1999), indicating that their mass assembly histories were likely different. M31 is generally assumed to be more massive, but the analysis of Watkins et al. (2010) argues that the two may have very similar halo masses. M31 is thought to be an earlier type spiral, but Beaton et al. (2007) revealed M31 to have a boxy bulge, indicative of a bar, and making M31 a twin of the MW in that regard. Huxor et al. (2011) point out that M31 possesses a significant population of luminous and compact globular clusters (GCs) at large galactocentric radii without counterparts in the MW, and that M31 also has a number of extended GCs, many of which are far larger than those in the MW. They suggest that the differences between the two GC systems could be, at least partly, explained by the differing accretion histories that M31 and MW have experienced. M31 has a higher stellar mass and appears to have undergone more late-time satellite accretion than the MW (e.g., Brown et al. 2006; Bernard et al. 2012; Deason et al. 2013; Bernard et al. 2015a, 2015b; Williams et al. 2015).

The differences between M31 and the MW may extend to their satellite populations. The presence of the true $\mathrm{dE}$ galaxy M32, a relatively rare occurrence in nature (see, e.g., Kormendy \& Bender 2012), indicates that something special has taken place in Andromeda's satellite history, but exactly what remains a topic for debate. The SFH for M32 derived by Monachesi et al. (2012) shows a nearly constant star formation rate (SFR) up until 2 Gyr ago producing stars with nearly solar metallicities.

Another possible difference is the presence of the more luminous dSphs NGC 147, NGC 185, and NGC 205. At $M_{V}=-14.6,-14.8$, and -16.5 , respectively, they are one to three magnitudes brighter than the MW's brightest dSphs, Fornax and Sagittarius, at $M_{V}=-13.4$ and -13.5 (McConnachie 2012, though as a tidally disrupting galaxy, the luminosity for Sagittarius may represent a lower limit). This may be an indication of something significantly different in the formation of dSphs, or it may simply be the natural extension to higher luminosities in a more abundant population. Geha et al. (2015) have produced SFHs for NGC 147 and NGC 185 and found NGC 147 to have continued to produce stars well into intermediate ages, while NGC 185 contains mostly older stars. However, the direct interpretation of these SFHs is complicated by the positions of the observed fields beyond the half-light radii; the extremities often show predominantly older stars even in actively star-forming dwarfs. So the apparent surprise is the extended nature of the star formation in the outer regions of NGC 147. Note also that NGC 147 differs from NGC 185 in that NGC 147 shows the effects of a recent interaction (Crnojević et al. 2014).

Before this project, there were hints of possible differences between the M31 and MW dSphs. For example, the M31 dSphs present redder horizontal branch (HB) morphologies when compared to the MW dSphs (e.g., da Costa et al. 1996, 2000, 2002; McConnachie et al. 2007). Additionally, McConnachie \& Irwin (2006b) showed that the M31 dSphs generally have larger half-light radii than the MW dSphs. This was later 
Table 1

Summary of the ISLAndS Sample and Observations

\begin{tabular}{|c|c|c|c|c|c|c|c|c|c|c|}
\hline $\begin{array}{l}\text { Galaxy } \\
\text { (1) }\end{array}$ & $\begin{array}{c}H S T \\
\text { ID } \\
(2)\end{array}$ & $\begin{array}{c}\text { F475W } \\
\text { (s) } \\
\text { (3) }\end{array}$ & $\begin{array}{c}\text { F814W } \\
\text { (s) } \\
\text { (4) }\end{array}$ & $\begin{array}{c}(m-M)_{0} \\
(\mathrm{mag}) \\
(5)\end{array}$ & $\begin{array}{c}E(B-V) \\
(\mathrm{mag}) \\
(6)\end{array}$ & $\begin{array}{c}M_{V} \\
(\mathrm{mag}) \\
(7)\end{array}$ & $\begin{array}{c}\mathrm{R}_{1 / 2} \\
(\mathrm{pc}) \\
(8)\end{array}$ & $\begin{array}{c}D_{\mathrm{M} 31} \\
(\mathrm{kpc}) \\
(9)\end{array}$ & $\begin{array}{c}V_{c, 1 / 2} \\
\mathrm{~km} \mathrm{~s}^{-1} \\
(10)\end{array}$ & $\begin{array}{c}V_{c, 1 / 2} \\
\mathrm{~km} \mathrm{~s}^{-1} \\
(11)\end{array}$ \\
\hline And I & 13739 & 28,996 & 22,968 & 24.51 & 0.047 & -12.0 & 895 & 46 & $16.1 \pm 4.4$ & $18 \pm 4$ \\
\hline And II & 13028 & 22,472 & 17,796 & 24.17 & 0.054 & -12.6 & 1045 & 168 & $12.3 \pm 2.6$ & $\ldots$ \\
\hline And III & 13739 & 28,996 & 22,968 & 24.38 & 0.050 & -10.1 & 421 & 81 & $14.7 \pm 3.7$ & $16 \pm 2$ \\
\hline And XV & 13739 & 22,443 & 17,773 & 24.43 & 0.041 & -8.4 & 282 & 95 & $6.3_{-3.3}^{+3.4}$ & $7 \pm 3$ \\
\hline And XVI & 13028 & 17,194 & 13,622 & 23.71 & 0.059 & -7.6 & 151 & 263 & $8.8_{-2.7}^{+3.2}$ & $7 \pm 6$ \\
\hline And XXVIII & 13739 & 26,360 & 20,880 & 24.45 & 0.080 & -8.8 & 268 & 372 & $10.4_{-5.8}^{+7.7}$ & $8 \pm 3$ \\
\hline
\end{tabular}

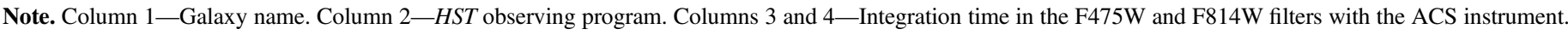

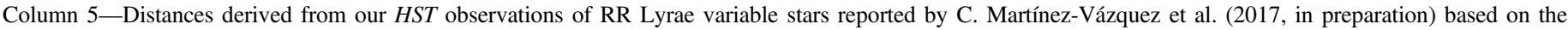

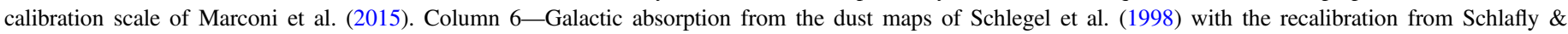

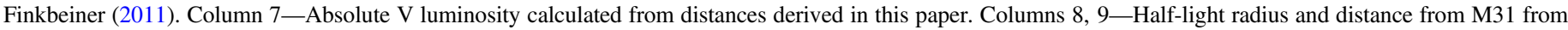

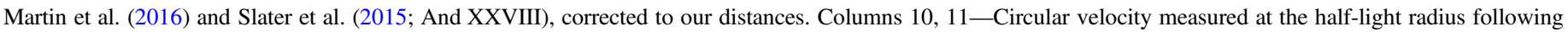
Walker et al. (2009) from Collins et al. (2014) and Tollerud et al. (2014).

quantified as differences in the mean scaling relations at the 1-2 $\sigma$ level (Brasseur et al. 2011; Tollerud et al. 2012; Collins et al. 2014). Regardless, the M31 dSphs occupy regions of parameter space for which there are no analogous MW systems, emphasizing that a full understanding of the origins of dwarf galaxy properties cannot be obtained from the MW system alone.

The early SFHs may possibly reflect the effects of the epoch of reionization. The realization that the reionization of the universe is quite inhomogeneous (e.g., Songaila 2004; Fan et al. 2006; Becker et al. 2015) has led to a better appreciation of the impact of the primary galaxy on the evolution of its satellites (e.g., Weinmann et al. 2007; Busha et al. 2010; Ocvirk \& Aubert 2011). For example, simulations by Mayer et al. $(2006,2007)$ show that the local ionizing radiation from the primary galaxy controls the temperature evolution and ionization state of the gas in the satellite dwarfs. This sets the efficiency of mass stripping by tides and gas removal by ram pressure from the dwarf satellites. The local UV flux, at the distance of a typical MW satellite, is estimated to have been more than an order of magnitude higher than the average cosmic UV background radiation at $z>1$. At that epoch, the primary galaxy was undergoing massive star formation at levels comparable to present-day starburst galaxies (Governato et al. 2007). Since the intensity and temporal evolution of the radiation field of the primary galaxy will depend on its SFH and mass assembly history, given the differences between the MW and M31, we might expect the evolution of their satellite galaxies to have been significantly different.

The structure of the paper is as follows. The ISLAndS sample is described in Section 2. The observations and data reduction are presented in Section 3. The SFHs of the sample galaxies are presented in Section 4. A comparison of the quenching times for the sample galaxies is given in Section 5. A comparison of the properties of the sample galaxies within and outside of the thin plane identified by Ibata et al. (2013) is presented in Section 6. Finally, we make our first attempts at our main goal, comparing the M31 and MW satellites in Section 7. The main conclusions of the work are summarized in Section 8. In this work, cosmological parameters of $H_{0}=67.8 \mathrm{~km} \mathrm{~s}^{-1} \mathrm{Mpc}^{-1}, \Omega_{m}=0.308$, and a flat universe with $\Omega_{\Lambda}=1-\Omega_{m}$ are assumed (i.e., Planck Collaboration et al. 2016).

\section{The ISLAndS Representative Sample}

\subsection{Properties of the ISLAndS Galaxies}

M31 has a diverse satellite galaxy population (e.g., McConnachie \& Irwin 2006a), and the number continues to grow with the discovery of increasingly fainter galaxies (e.g., McConnachie et al. 2008; Bell et al. 2011; Richardson et al. 2011; Slater et al. 2011; Martin et al. 2013a, 2013b). For the ISLAndS program, we are focusing on the M31 dSphs more luminous than $M_{V} \lesssim-7$ in order to derive well-constrained SFHs for comparison to their MW analogues. These galaxies are massive enough that their status as galaxies is not controversial, and they are populous enough to provide strong constraints on their SFHs over the age of the universe. The abundance of M31 companions allows us to design a representative sample-which spans the range of properties of the ensemble-yet consists of the galaxies that are least expensive to observe.

Our sample of six galaxies is presented in Table 1, and has been selected by balancing exposure time considerations with the requirement to observe galaxies spanning a range of luminosity $\left(M_{V}\right)$, half-light radius $\left(R_{H}\right)$, and distance from M31 $\left(D_{\mathrm{M} 31}\right)$. Thus, the sample consists of galaxies with minimal distances from us and minimal foreground extinction. Because of the large angular size of the M31 satellite distribution and the relatively low Galactic latitude of M31 $\left(b=-21^{\circ} .6\right)$, there is a large range in foreground reddening to the satellites $(0.04 \leqslant E(B-V) \leqslant 0.20$ McConnachie 2012). Thus, there is a significant range in required exposure times for the M31 satellites to be observed to the required depth.

The positions in the sky of the ISLAndS sample are shown in Figure 1. Figure 1 immediately shows that the ISLAndS sample spans a large range in projected distance and the true distances from M31 range from 58 to $370 \mathrm{kpc}$ (Table 1). Based on "fragmentary" data, van den Bergh (1994b) first pointed out the general trend for the stellar content of faint Local Group dwarfs to correlate with distance from the Galaxy. This correlation is in the sense that the closest have predominantly old stellar populations while the more distant have larger intermediate-age populations. While not without exceptions, modern observations have shown that this trend is still valid. A goal of our program is to determine whether the M31 dSphs show a similar trend of increasing mean age with distance from 


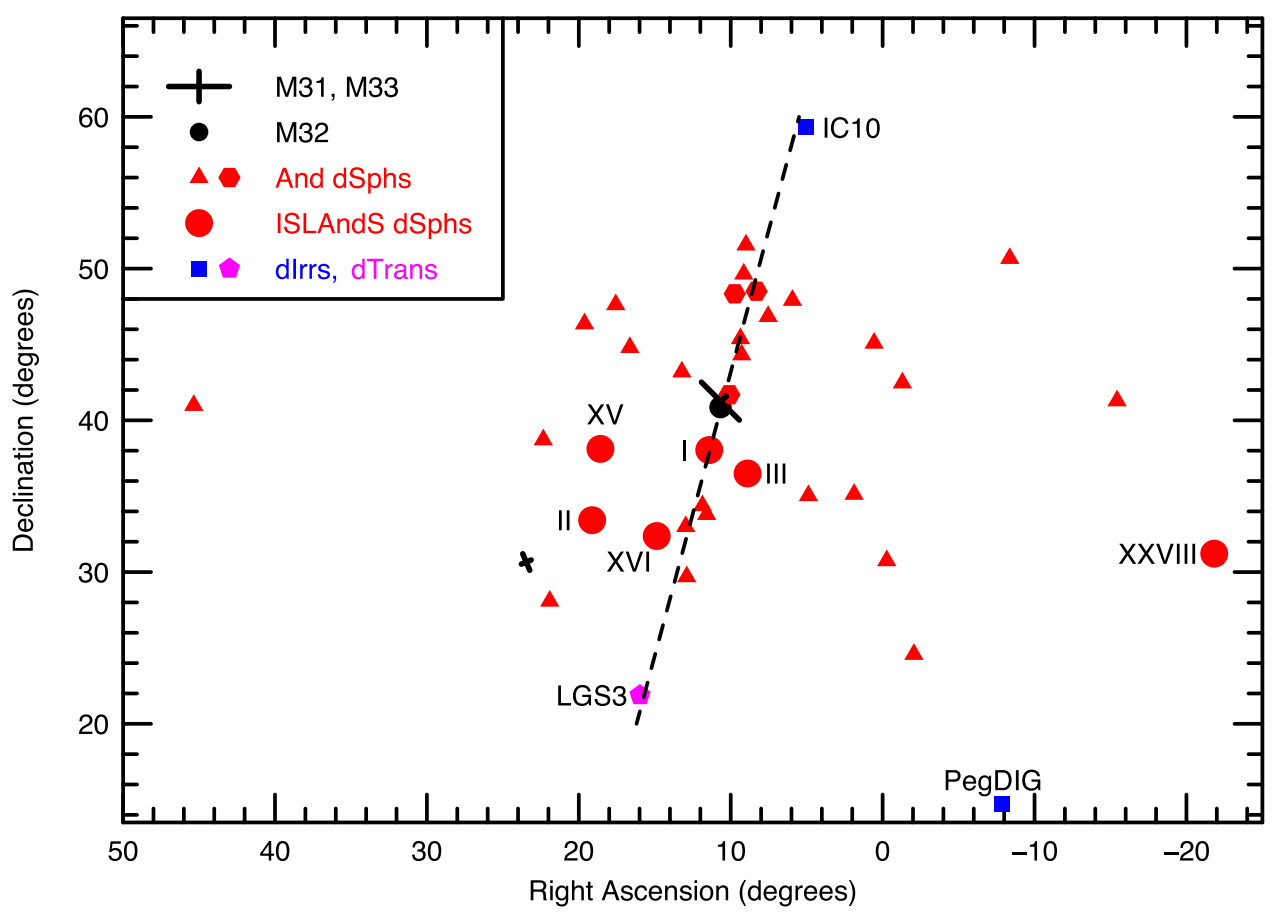

Figure 1. Companions to Andromeda are plotted showing their positions in the sky relative to M31 and M33. The three high luminosity dSphs (NGC 147, NGC 185, and NGC 205) are distinguished from the rest of the dSphs with hexagon symbols. The positions of the ISLAndS sample are highlighted with larger symbols and labels. Note that the ISLAndS sample galaxies span a large range in distance from M31. The dashed line represents the approximate position of the thin plane discovered by Ibata et al. (2013). Note that three galaxies from the ISLAndS sample (And I, And III, and And XVI) are located in the thin plane and three (And II, And XV, and And XXVIII) are outside of the plane.

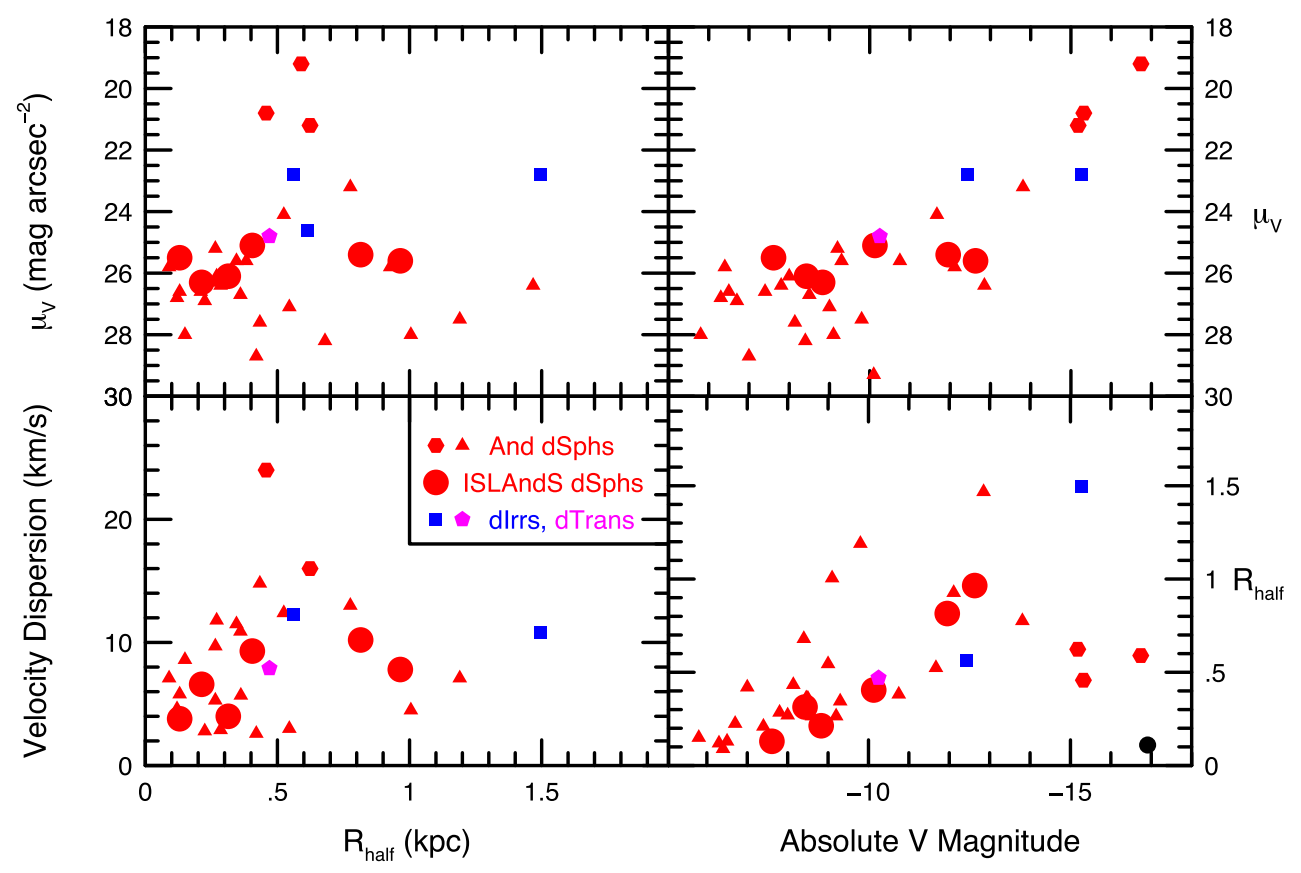

Figure 2. Following Kormendy (1985), a diagram showing the intrinsic properties (absolute $V$ magnitude, half-light radius, central $V$ surface brightness, and stellar velocity dispersion) of the M31 satellites. The symbols are identical to those used in Figure 1. The distributions of the ISLAndS sample relative to the rest of the M31 dSph companions show that the ISLAndS sample is representative with the exception of the lack of very low luminosity dSphs. The lack of the lowest central surface brightness objects is a result of the lower limit on luminosity so that the number of observed stars would be large enough to provide sufficient constraints on the history of the earliest star formation and the correlation of central surface brightness with luminosity. M32 does not appear in three of the panels due to its high central surface brightness (11 mag $\left.\operatorname{arcsec}^{-2}\right)$ and velocity dispersion $\left(92 \mathrm{kms}^{-1}\right)$.

the host as shown by the Galactic dSphs. Additionally, half of the sample are in the thin plane of co-rotating galaxies identified by Ibata et al. (2013).
In Figure 2, we show a "Kormendy" diagram (Kormendy 1985) presenting the intrinsic properties of all of the M31 satellites and highlighting the ISLAndS sample. This 


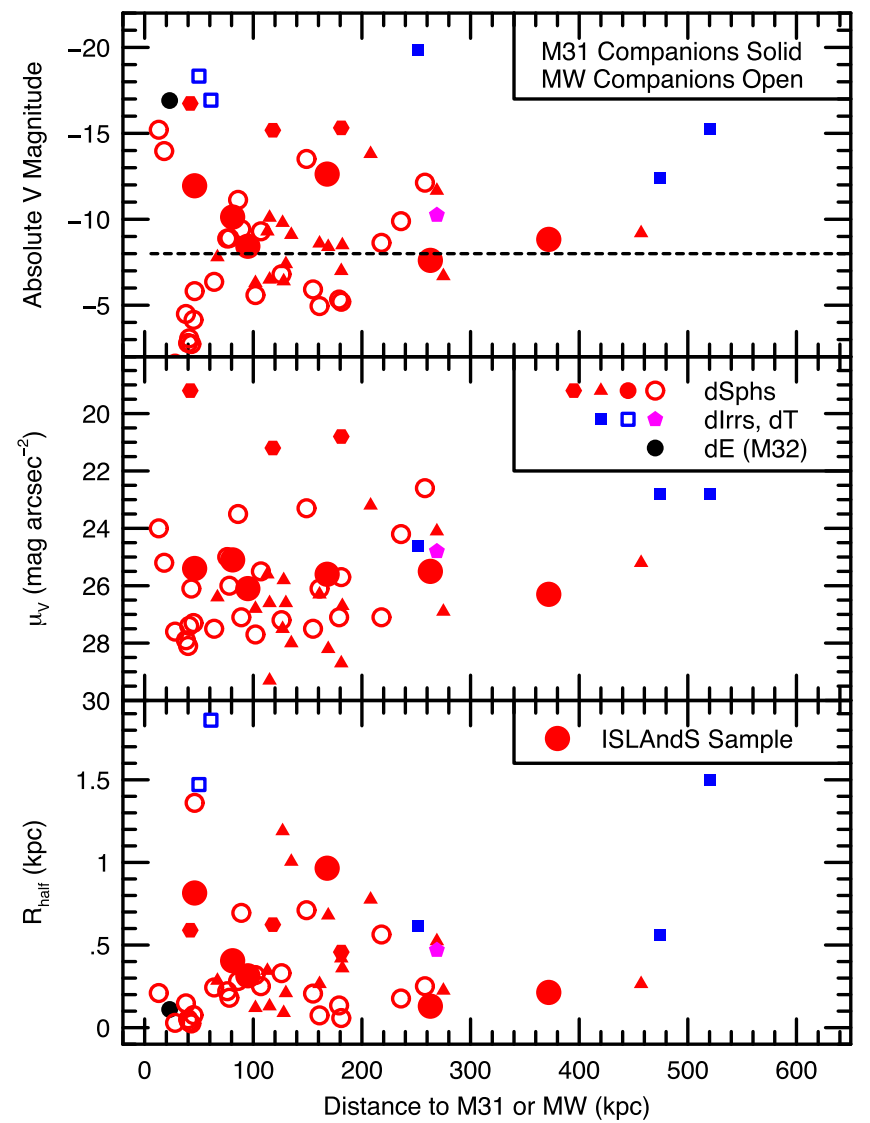

Figure 3. Properties of companions to Andromeda (luminosity, central surface brightness, and half-light radius) as a function of the distance to Andromeda highlighting the positions of the ISLAndS sample observed here. For comparison, the satellites of the MW are added. The dashed line in the upper plot indicates the lower limit for the ISLAndS sample to provide sufficiently populous color-magnitude diagrams for strong constraints on the SFHs. Note that M32 is well off the scale of the middle panel of this plot due to its high central surface brightness of $11.1 \mathrm{mag} \operatorname{arcsec}^{-2}$.

figure was assembled from data in McConnachie (2012), updated with data from Conn et al. (2012) and Martin et al. (2016), and the data in Table 1. As can be seen in Figure 2, the ISLAndS sample galaxies give a good representative coverage of the range of intrinsic properties of the M31 dSph satellites. The exception is a lack of very low surface brightness galaxies in the ISLAndS sample. This is a result of the lower limit on luminosity imposed in order to obtain strong constraints on the SFHs.

In Figure 3, we have plotted a comparison of the half-light radii, central surface brightnesses, and luminosities of the M31 and MW satellites as a function of distance from the host galaxy. Figure 3 shows that the ISLAndS M31 dSphs span similar ranges in luminosity and radial distance from host as the well-studied MW dSphs. These true three-dimensional separations are based on differential heliocentric distances (see McConnachie et al. 2004, 2005; McConnachie \& Irwin 2006a; Conn et al. 2012; McConnachie 2012).

Phase I of this project (HST cycle 20, observed 2013 November) started with observations of And II and And XVI (see Weisz et al. 2014d). Cycle 20 Hubble Space Telescope proposals for targets in the restricted RA zone around Andromeda were limited to a total of 30 orbits, so we proposed the two galaxies that could be done in 30 orbits. Our cycle 22 program allowed us to extend our radial coverage to smaller
(And I and And III) and larger radii (And XXVIII) allowing a direct comparison of inner versus outer galaxies and also to fill in the middle in the $M_{V}, R_{H}$ plane. And XXVIII and And XV also address the critical question of whether the young mean age of And XVI is an anomaly or shows the importance of separation distance over mass for early-quenching (see discussions in Weisz et al. 2014d; Monelli et al. 2016). Together, these six galaxies allow us to test the hypothesis that differences between MW and M31 dSphs are due to the early evolution of the parent galaxy.

\subsection{Kinematic and Abundance Data from the Literature}

In Table 2, we have assembled data from the literature for the kinematics and chemical abundances for the ISLAndS sample galaxies. These data come from spectroscopic studies of individual RGB stars in these galaxies and represent a significant investment of ground-based (especially Keck) observing time. We have two reasons for the compilation in Table 2. First, we would like to present the original sources for reference for the masses and chemical abundances for discussion of the ISLAndS galaxies. Second, while there has been tremendous progress on this front, we would like to highlight that more work is still needed. In many regards, And II is the ideal example of what can be learned. With hundreds of spectra observed, the velocity dispersion is very well defined, in fact, the large number of spectra allowed Amorisco et al. (2014) to identify a kinematically cold component in And II. Note that there is no overlap with our HST fields of view and the kinematically cold component in And II, and, to date, this component has not been observed with the HST in order to study its SFH.

Spectroscopic chemical abundances also hold promise for a better understanding of the evolution of these galaxies. Spectroscopic stellar abundances provide a way to increase the precision in determining the SFHs at early ages where the color-magnitude diagram technique has challenging time resolution limitations (de Boer et al. 2012a, 2012b, 2014; Brown et al. 2014; Dolphin 2016). The improved early time resolution of these SFHs arises because the spectroscopic abundances constrain potential degeneracies between age and metallicity. However, some care needs to be exercised because, to date, these techniques have not been demonstrated using multiple stellar libraries. Since the dominant uncertainty in deriving SFHs is the systematic uncertainty of choosing a stellar evolution library (see the Appendix), we regard these improvements in time resolution with a degree of skepticism (see discussion in Dolphin 2016). Regardless, ambitious spectroscopic abundance studies primarily hold the promise for significantly more reliable age-metallicity relationships (AMRs) and these are vital to a complete understanding of the evolution of these galaxies (Dolphin 2016). Additionally, as pointed out in McConnachie \& Côté (2010), multiple epochs are highly desired to eliminate inflated velocity dispersions due to binaries.

Given the value of the investment of HST observing time dedicated to the study of the ISLAndS sample, we heartily encourage additional ground-based spectroscopic campaigns to bring the other galaxies up to the remarkable standard achieved for And II. We note especially the near absence of relative chemical abundances (e.g., $[\alpha / \mathrm{Fe}]$ ). Dotter et al. (2007) has emphasized the important role that accurate relative chemical abundances can play in deriving SFHs. 
Table 2

ISLAndS Sample Kinematic and Abundance Observations from the Literature

\begin{tabular}{|c|c|c|c|c|c|c|c|c|}
\hline $\begin{array}{l}\text { Galaxy } \\
\text { (1) }\end{array}$ & $\begin{array}{c}\sigma_{r v} \\
\left(\mathrm{~km} \mathrm{~s}^{-1}\right) \\
(2)\end{array}$ & $\begin{array}{l}\text { N Stars } \\
\text { (3) }\end{array}$ & $\begin{array}{c}\langle[\mathrm{Fe} / \mathrm{H}]\rangle \\
(4)\end{array}$ & $\begin{array}{c}\sigma_{[\mathrm{Fe} / \mathrm{H}]} \\
(5)\end{array}$ & $\begin{array}{l}\text { N Stars } \\
\text { (6) }\end{array}$ & $\begin{array}{c}\langle[\alpha / \mathrm{Fe}]\rangle \\
(7)\end{array}$ & $\begin{array}{c}\text { N Stars } \\
\text { (8) }\end{array}$ & $\begin{array}{l}\text { References } \\
\text { (9) }\end{array}$ \\
\hline And I & $10.6 \pm 1.1$ & 80 & $-1.45 \pm 0.04$ & 0.37 & 80 & $\cdots$ & $\cdots$ & Kalirai et al. (2010) \\
\hline And I & $10.2 \pm 1.9$ & 51 & $\cdots$ & $\cdots$ & $\cdots$ & $\cdots$ & $\cdots$ & Tollerud et al. (2012) \\
\hline And I & $8.2 \pm 1.7$ & 49 & $\ldots$ & $\ldots$ & $\ldots$ & $\ldots$ & $\ldots$ & Tollerud et al. (2012) \\
\hline And I & $\cdots$ & $\cdots$ & $-1.11 \pm 0.12$ & $\cdots$ & 31 & $0.28 \pm 0.16$ & 7 & Vargas et al. (2014) \\
\hline And II & $7.3 \pm 0.8$ & 95 & $-1.64 \pm 0.04$ & 0.34 & 95 & $\cdots$ & $\cdots$ & Kalirai et al. (2010) \\
\hline And II & $7.8 \pm 1.1$ & 531 & $-1.39 \pm 0.03$ & $0.72 \pm 0.03$ & 477 & $\cdots$ & $\cdots$ & Ho et al. (2012) \\
\hline And II & $\cdots$ & $\cdots$ & $-1.37 \pm 0.12$ & $\cdots$ & 248 & $0.03 \pm 0.09$ & 56 & Vargas et al. (2014) \\
\hline And II & $\ldots$ & $\cdots$ & $-1.25 \pm 0.05$ & $0.49 \pm 0.04$ & 300 & $\cdots$ & $\cdots$ & Ho et al. (2015) \\
\hline And III & $4.7 \pm 1.8$ & 43 & $-1.78 \pm 0.04$ & 0.27 & 43 & $\cdots$ & $\ldots$ & Kalirai et al. (2010) \\
\hline And III & $9.3 \pm 1.4$ & 62 & $\cdots$ & $\cdots$ & $\cdots$ & $\cdots$ & $\cdots$ & Tollerud et al. (2012) \\
\hline And III & $\cdots$ & $\cdots$ & $-1.81 \pm 0.12$ & $\cdots$ & 35 & $0.33 \pm 0.21$ & 8 & Vargas et al. (2014) \\
\hline And XV & $4.0 \pm 1.4$ & 29 & $\cdots$ & $\cdots$ & $\cdots$ & $\cdots$ & $\cdots$ & Tollerud et al. (2012) \\
\hline And XVI & $3.8 \pm 2.9$ & 7 & $\cdots$ & $\cdots$ & $\cdots$ & $\cdots$ & $\cdots$ & Tollerud et al. (2012) \\
\hline And XVI & $5.8_{-0.9}^{+1.1}$ & 20 & $-2.0 \pm 0.1$ & $\cdots$ & 12 & $\cdots$ & $\cdots$ & Collins et al. (2015) \\
\hline And XXVIII & $4.9 \pm 1.6$ & 18 & $\sim-2.0$ & $\cdots$ & $\cdots$ & $\cdots$ & $\cdots$ & Tollerud et al. (2013) \\
\hline And XXVIII & $6.6_{-2.1}^{+2.9}$ & 17 & $-2.1 \pm 0.3$ & $\cdots$ & 17 & $\cdots$ & $\ldots$ & Collins et al. (2013) \\
\hline And XXVIII & $\cdots$ & $\cdots$ & $-1.84 \pm 0.15$ & $0.65 \pm 0.15$ & 13 & $\cdots$ & $\cdots$ & Slater et al. (2015) \\
\hline
\end{tabular}

Note. Column 1-Galaxy name. Column 2-Line-of-sight velocity dispersion. Column 3-Number of stars used to calculate the velocity dispersion. Column 4Average metallicity. Column 5-Dispersion in metallicity. Column 6-Number of stars used to calculate metallicity. Column 7-Average alpha element abundance relative to metallicity Column 8 -Number of stars used to calculate average alpha element abundance. Column 9-Reference for literature source.

\section{Observations and Data Reduction}

\subsection{The Observations}

The HST observations of the six M31 satellites were obtained between 2013 October and 2015 September. The two galaxies observed in cycle 20 (And II and And XVI) have previously been reported in Weisz et al. (2014d). All observations were reduced in a uniform manner, and a brief summary is provided here.

Following the observing protocols established by the Local Cosmology from Isolated Dwarfs (LCID) program (e.g., Monelli et al. 2010a, 2010b; Hidalgo et al. 2011), the F475W and F814W bands were selected as the most efficient combination to trace age differences at old ages, since they provide the smallest relative error in age and metallicity in the main-sequence and subgiant regions. Asymmetric exposure times were chosen such that the uncertainties in the two different photometry bands were equal at an F475W-F814W color of 1 -essentially the color of the main sequence one magnitude below the oMSTO.

The observations were organized into two orbit visits, and each orbit was split into one F475W and one F814W exposure in a sequence of F475W-F814W-F814W-F475W (in order to maximize sampling of variable star light curves). Each visit acquired $2363 \mathrm{~s}$ of integration time in F475W and $2088 \mathrm{~s}$ of integration time in F814W with the ACS. The total integration times are given in columns 3 and 4 of Table 1. Simultaneously, parallel fields (see Figure 4) were observed with the WFC3 camera with exposure times of $2759 \mathrm{~s}$ in F475W and $2322 \mathrm{~s}$ in F814W. Dithers of a few pixels between exposures were introduced to minimize the impact of pixel-to-pixel sensitivity variations ("hot pixels") in the CCDs. As with the LCID program, the visits were planned to take place over several days in order to properly sample the light curves for variable stars with periods less than approximately two days. Since it was anticipated that the bulk of the variable stars would be RR
Lyrae with periods of roughly 0.5 days, the scheduling of the visits was designed to minimize cadences of $12 \mathrm{hr}$ ( $\sim 8$ orbits) so that the observations would not all be taken near the same phase. This worked well for the LCID program (e.g., Bernard et al. 2009, 2010) and also for the present program (C. Martínez-Vázquez et al. 2017, in preparation).

The positions of the observed fields are shown in Figure 4. Here it can be seen that the ACS fields of And I and And II cover only part of the galaxy within the half-light radius and the parallel WFC3 fields contain many member stars. Some member stars were detected in the WFC3 field of And III, but not nearly enough to produce an SFH with reasonable uncertainties. For And XV, And XVI, and And XXVIII, the ACS field of view covers most of the galaxy out to the halflight radius and the WFC3 field is distant enough that a minimal number of member stars are expected.

\subsection{Data Reduction}

We analyzed images taken directly from the STScI pipeline (bias, flat-field, and image distortion corrected) working with the charge transfer efficiency corrected images (i.e., .flc images). Two PSF-fitting photometry packages, DAOPHOT/ ALLFRAME (Stetson 1994) and DOLPHOT, an updated version of HSTPHOT with ACS and WFC3 specific modules (Dolphin 2000), were used independently to obtain the photometry of the resolved stars. See Monelli et al. (2010a) for more details about both photometry reduction procedures. Individual photometry catalogs were calibrated using equations provided by the STScI (e.g., Sirianni et al. 2005) with the most recent updates (e.g., ACS ISR 12-01). Similar to our experience with the LCID program (Monelli et al. 2010a) the differences between the two sets of photometry are small (e.g., zero point offsets of $\sim 0.01$ to $0.02 \mathrm{mag}$ ) and typical for obtaining $H S T$ photometry with different methods (Hill et al. 1998; Holtzman et al. 2006). Thus, for simplicity, the rest of this paper is based on only the DOLPHOT photometry data sets. 


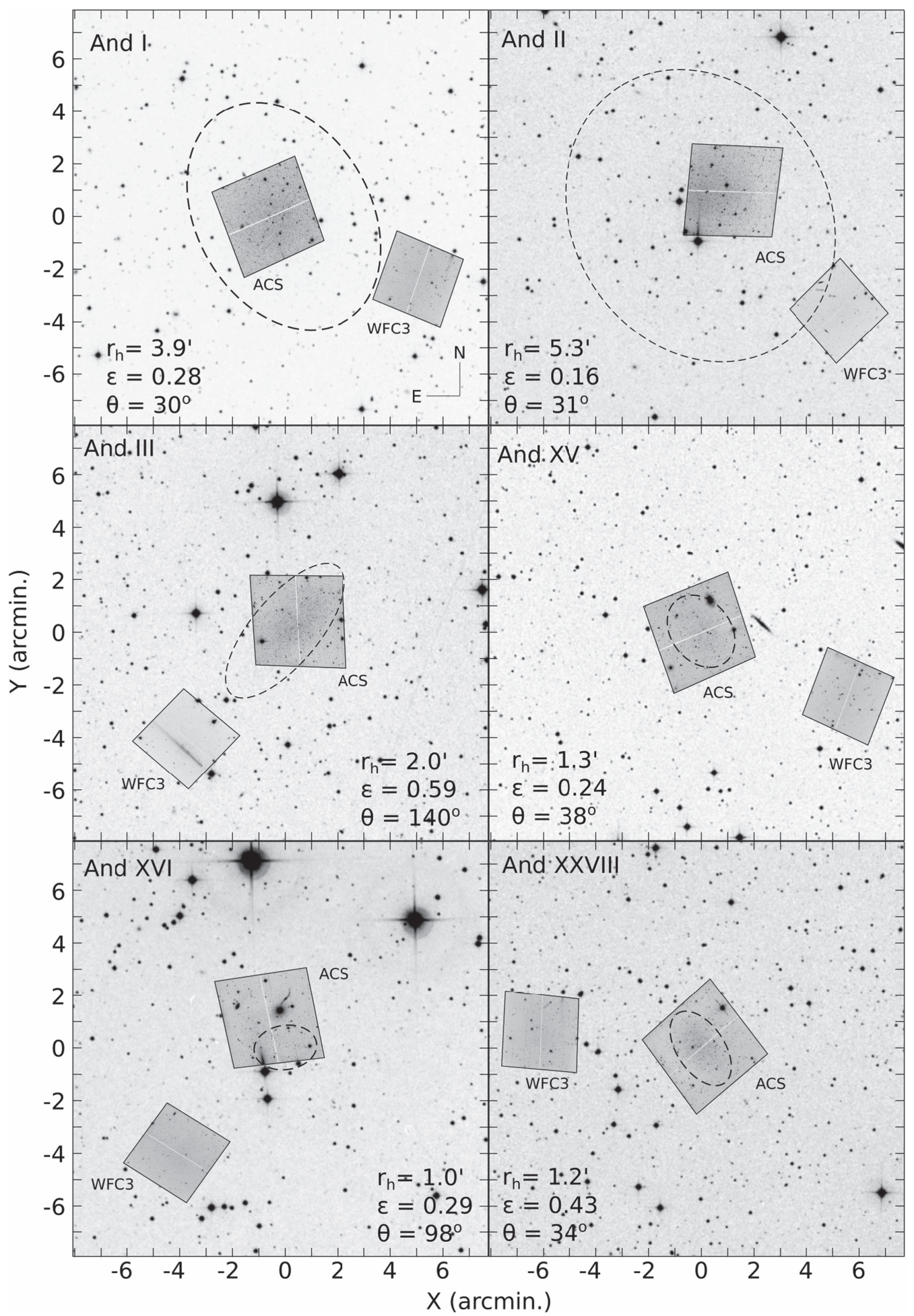

Figure 4. HST/ACS observing positions for the ACS and WFC3 cameras for the ISLAndS sample. The dashed circles show the half-light radii for the galaxies. The ACS field for And XVI was not centered in order to avoid a bright foreground star. The ACS fields for And I, And II, and And III were offset from the galaxy center to provide a better radial coverage.

Signal-to-noise limitations, detector defects, and stellar crowding can all impact the quality of the photometry of resolved stars with the resulting loss of stars, changes in measured stellar colors and magnitudes, and systematic uncertainties. To characterize these observational effects, we injected $\sim 10^{6}$ artificial stars in the observed images and obtained their photometry in an identical manner as for the real stars. Monelli et al. (2010a) and Hidalgo et al. (2011) provide detailed descriptions of the procedures we adopt for the characterization and simulation of these observational effects.

\subsection{The ACS Color-Magnitude Diagrams}

The CMDs for the ACS fields of the observed M31 satellites are shown in Figure 5. The left axis shows the observed F814W 


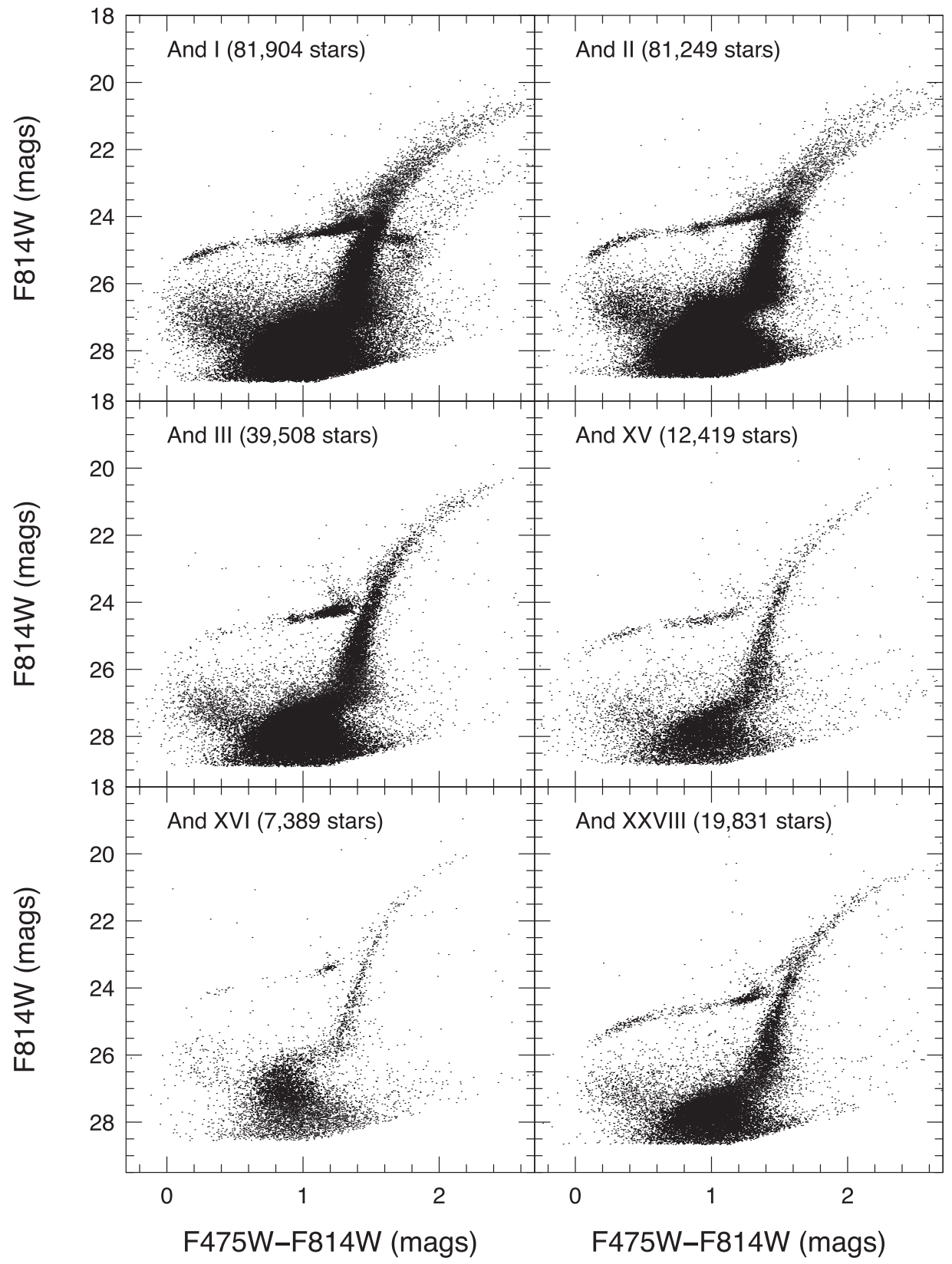

Figure 5. HST/ACS color-magnitude diagrams of the ISLAndS sample. The number of sources that passed the quality cuts is reported for each galaxy. In all cases, the photometry reaches to below the oldest main-sequence turnoffs. The CMD for And I shows contamination from Andromeda's Giant Stellar Stream (Ibata et al. 2001; Ferguson et al. 2002; McConnachie et al. 2003). The extra depth in And XVI is due to a preliminary distance measurement that was over-estimated.

magnitudes in the ACS photometric system uncorrected for extinction. Figure 5 shows that the ACS photometry reaches below the oMSTOs allowing for very strong constraints on the oldest epochs of star formation. These observations of And I, And II, and And III are $\sim 2.5 \mathrm{mag}$ fainter than the deepest CMDs previously obtained for these galaxies (da Costa et al. 1996, 2000, 2002).

There are several notable features that are common to all six ACS CMDs. All six galaxies show the presence of blue horizontal branch stars (BHBs). This is generally taken as evidence of the presence of stars with ages comparable to the MW GC population (see the discussion in Gallart et al. 2005). To date, all galaxies that have been observed with sufficient photometric depth have shown at least some star formation at the earliest times. da Costa et al. $(1996,2000,2002)$ noted that
And I, And II, and And III all had substantial populations of red horizontal branch stars (RHBs) and this was interpreted to mean that all three galaxies had substantial populations of stars younger than the typical MW GCs. A direct connection between HB morphology and SFH remains elusive. For example, the MW companions Draco and Ursa Minor have identical absolute magnitudes and very different HB morphologies. Draco has a predominantly red HB and Ursa Minor has a predominantly blue HB, yet SFHs derived from oMSTO photometry seem to indicate that Draco formed most of its stars before Ursa Minor (Olszewski \& Aaronson 1985; Grillmair et al. 1998; Mighell \& Burke 1999; Weisz et al. 2014a). Thus, while the HB morphology can provide clues to the old age SFHs (and metallicities), there is no substitute for oMSTO photometry. 
In fact, all six galaxies show complex HB morphologies, indicative of a metallicity or age range. In all six galaxies, the $\mathrm{HB}$ is extended from the blue to the red side, with the population of RR Lyrae variable stars noticeable as a clump at $(\mathrm{F} 475 \mathrm{~W}-\mathrm{F} 814 \mathrm{~W}) \sim 0.9$ (in some cases spanning a wider magnitude range than the HB stars at either side). The relative populations of the blue and red HBs varies between galaxies, with And I, And II, and And XXVIII having both the blue and the red HBs well populated, while And III and And XVI have more prominent red HBs. In And I and And II the red HBs even merge with the RGBs, which are very wide, indicating a wide metallicity range.

All six galaxies also show a significant blue plume in the CMDs below the HB and above the oldest MS turnoffs. These populations are typically identified as a "blue straggler star" (BSS) population. While first observed in the GC M3 (e.g., Sandage 1953), BSSs are found in GCs, populous open clusters, and sufficiently populous dSphs. Several scenarios can give rise to a BSS, but most are thought to originate from close binaries arising from either collisional binaries (favored in the dense environments of the centers of GCs; e.g., Meylan \& Heggie 1997) or primordial binaries (likely ubiquitous). The binary nature of BSSs has been observed directly (e.g., Mateo et al. 1990) and there is chemical evidence to support this scenario (e.g., Ferraro et al. 2006). Note also the recent work by Gosnell et al. (2014, 2015) finding direct evidence of mass transfer in the BSS population of the MW open cluster NGC 188. The presence of BSSs presents a true ambiguity in reconstructing an SFH from a CMD (e.g., Hurley-Keller et al. 1998; Aparicio et al. 2001; Carrera et al. 2002). If these stars are interpreted as MS stars, they have ages indicating star formation as recent as $\sim 2 \mathrm{Gyr}$ ago. However, these populations are ubiquitous in $\mathrm{dSph}$ galaxies (at low levels), and there are good arguments that they are due to the altered evolution of coalescing or mass-transferring primordial binary stars (see the discussions in Mapelli et al. 2007, 2009; Momany et al. 2007; Monelli et al. 2012; Santana et al. 2013). However, to date, no one-to-one relationship has been proposed for the ratio of old age stellar populations to BSSs, so the possibility exists that in some dSph galaxies there could be younger MS stars mixed in with (or, equivalently, hidden by) the BSS population. In a future study, we hope to be able to use the ISLAndS sample to produce a quantitative measure of the close binary fraction necessary to produce the BSS populations in dSph galaxies.

All six galaxies also show, to differing degrees, the presence of an upward extension to the HB. In models, this part of the color-magnitude diagram is populated by helium burning stars with ages between $\sim 2-4$ Gyr. Helium burning stars with even younger ages can also occupy this region, but they would be accompanied by a significant population of bright MS stars, which we do not see in the present CMDs. Note that in the BSS interpretation, there is an upper limit to the MS stellar mass at twice the mass of the stars at the turnoff corresponding to the quenching time. At a constant metallicity, the positions of these intermediate-age red clump stars form a spur in the $\mathrm{CMD}$, which initially increases in luminosity and color (getting redder) with decreasing age, and then continues to increase in luminosity but turns bluer with even younger ages. These spurs off of the red clump are displayed prominently in the CMDs of Leo A (Cole et al. 2007), IC 1613 (Skillman et al. 2014), and Aquarius (DDO 210; Cole et al. 2014) because of the continuous star formation in these galaxies. The presence of these spurs in the ISLAndS dSphs is likely a reflection of the BSS population already noted. The detailed modeling presented in the next section shows that the ratio of BSSs to these stars is consistent with this scenario. There is one feature that is lacking in all six galaxies, and that is obvious, distinct multiple generations of star formation as evidenced by multiple sequences in the subgiant region. This is not a common feature of dSph galaxies, but it is seen famously in the CMD of the MW companion Carina, where early photometry showed a broad distribution of stars in the subgiant region, which was correctly interpreted as due to multiple episodes of star formation (Hurley-Keller et al. 1998). Deeper observations of Carina, comparable to the CMDs presented here (Monelli et al. 2003; Bono et al. 2010; Weisz et al. 2014a), showed distinctly separate subgiant branches. In this regard, the SFH and chemical evolution of Carina has produced a unique CMD.

There are other features of the individual galaxy CMDs worth noting. The CMD of And I is contaminated by Andromeda's Giant Stellar Stream (Ibata et al. 2001; Ferguson et al. 2002; McConnachie et al. 2003). This stream is made up of relatively metal-rich stars and is consistent with a relatively recent tidal origin, now thought to be the result of the complete disruption of an infalling satellite by M31 (Ibata et al. 2004; Fardal et al. 2013; Sadoun et al. 2014). This stream is located $\sim 100 \mathrm{kpc}$ behind And I, and thus, features are easily visually separated from And I in the CMD. The stream's RGB appears redder and fainter than that of And I and has a prominent red clump also redder than the RGB of And I.

The bifurcated RGB in And II is another notable feature in Figure 5. This feature was first noted by da Costa et al. (2000) and attributed to distinct populations in metallicity. McConnachie et al. (2007) noted that the stars of the red branch of the RGB were centrally located, adding further evidence for two distinct populations. Weisz et al. (2014d) have previously presented this CMD of And II and work is in progress to combine wide-field ground-based imaging along with our ACS and WFC3 observations to explore spatial variations in the populations of And II.

The photometry of And XVI is deeper than those of the other galaxies. This is because, when the observations were taken, the distance to And XVI was thought to be slightly larger than the accepted value today. As a result, the luminosity of And XVI is less than originally thought and the CMD is correspondingly less populated than originally anticipated.

\subsection{The WFC3 Parallel Fields}

For all six ISLAndS galaxies, parallel observations were obtained with the WFC3 camera in F475W and F814W (see Figure 4). In three of the galaxies (And XV, And XVI, and And XXVIII), there were essentially no detectable stellar populations in the WFC3 fields.

Figure 6 shows the CMDs for the WFC3 parallel fields in And I, And II, and And III. In And I, the WFC3 CMD appears to be very similar to that of the ACS except that it is much less populous. The giant stellar stream is more prominent relative to And I, as would be expected. In And II, only the older, metalpoor RGB is present (brighter and bluer); there is no evidence of the second RGB, which indicates that the younger, more metal-rich stars are more centrally concentrated. In And III, the stellar population is detectable, but there are insufficient stars for strong constraints on the SFH. The SFHs of the outer fields 


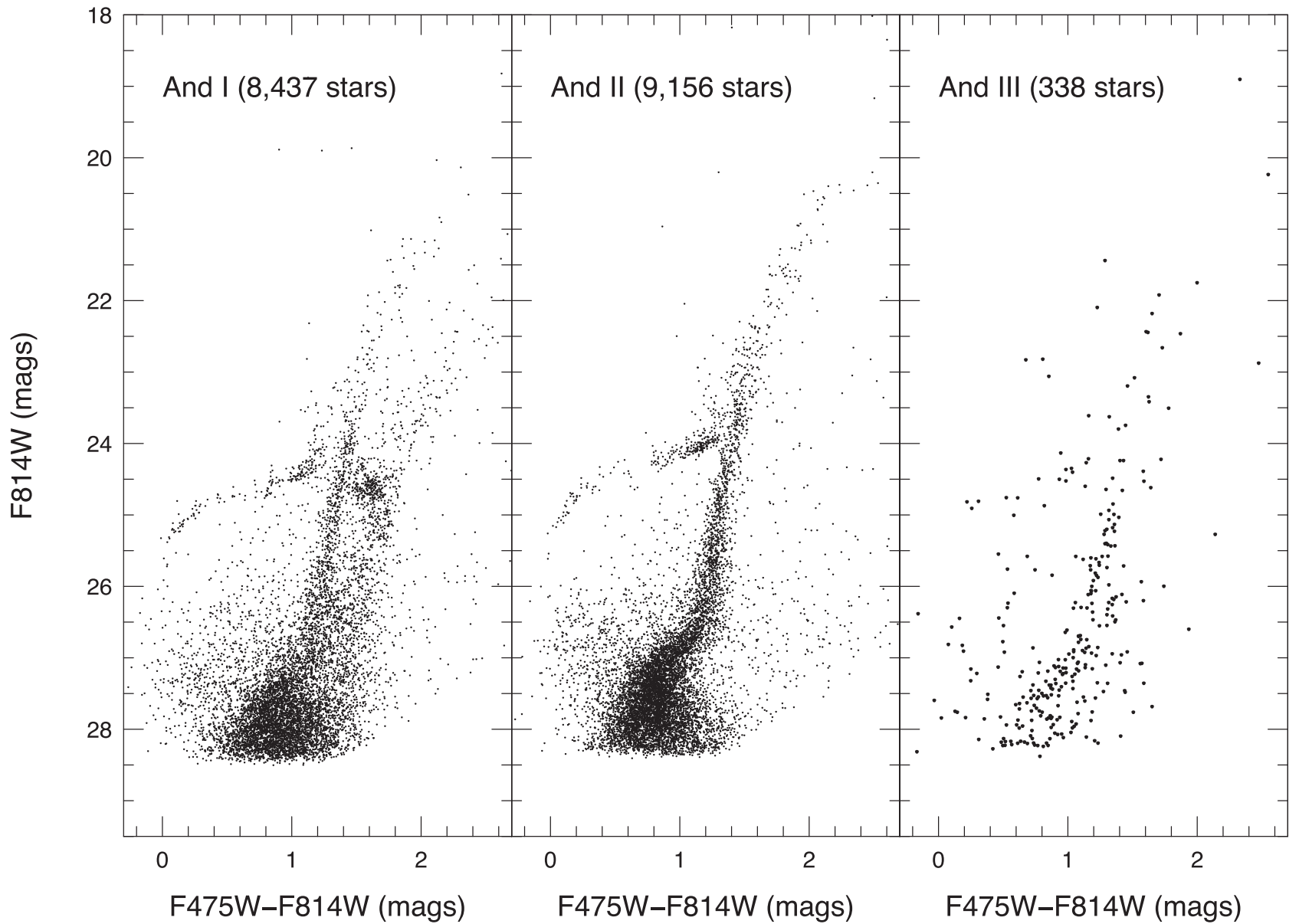

Figure 6. HST/WFC3 color-magnitude diagrams of the ISLAndS sample. The number of sources that passed the quality cuts are reported for each galaxy. In both cases, the photometry reaches the oldest main sequence turnoffs, but is not as deep as the ACS photometry. Because of its larger radius from the galaxy, the WFC3 CMD for And I is dominated by contamination from Andromeda's Giant Stellar Stream (Ibata et al. 2001; Ferguson et al. 2002; McConnachie et al. 2003).

will not be presented here. These fields will be used for detailed studies of the radial gradients in stellar populations for these galaxies. The WFC3 CMDs are presented here for completeness.

\section{THE SFHs of M31 Satellites}

\subsection{Distances}

The ultimate goal of the ISLAndS project is a detailed comparison of the early SFHs of M31 and MW satellite galaxies. For a reliable comparison of the earliest SFHs of MW and M31 satellites, a uniform distance measurement methodology is required. Note that systematic uncertainties in distance estimates can play a significant role in the determination of the SFRs at the earliest times (ages $\geqslant 5 \mathrm{Gyr}$ ), where a $\sim 0.1 \mathrm{mag}$ difference in MSTO luminosity corresponds to a $\sim 1 \mathrm{Gyr}$ difference in age. Also note that in deriving SFHs, it is quite common to treat the distance as a free parameter, which allows for minimizing differences between the observations and the model CMDs produced from stellar libraries (e.g., Dolphin 2012, 2016). However, since the derived ages of the oldest stars are very sensitive to distances, it is mandatory to use distances from a self-consistent distance scale, and best if the galaxy distances are determined independently from their SFHs. The sharp photometric edge of the tip of the RGB (TRGB) in the most populous CMDs allows very accurate distance determinations (e.g., Rizzi et al. 2007). However, in the lower luminosity galaxies, the TRGB is less well populated, and this can lead to a bias toward overestimating the distance (e.g., as discussed in McConnachie et al. 2004; Makarov et al. 2006; Conn et al. 2011, 2012; McQuinn et al. 2013). Some of the galaxies that we wish to compare are in the regime where TRGB distances become liable to these larger uncertainties. Thus, we choose to base our distances on observations of RR Lyrae stars.

C. Martínez-Vázquez et al. (2017, in preparation) present a detailed study of the variable stars in the ISLAndS galaxies. They derive distances for each of the galaxies using various methods, but favor distances based on RR Lyrae stars. Specifically, they derive distances using the theoretical period-Weisenheit relations (PWR) calculated by Marconi et al. (2015). Using the PWRs allows very accurate distances because they are, by construction, independent of reddening and they account for the position of the object within the instability strip. Additionally, the observational studies of RR Lyrae stars in Local Group galaxies continues to grow, so that a common distance scale is possible over much of the Local Group (see the discussion in C. Martínez-Vázquez et al. 2017, in preparation). C. Martínez-Vázquez et al. (2017, in preparation) compare the distances derived from the PWRs with other distance determination methods using the RR Lyrae stars and TRGB distance determinations and find overall good consistency. For example, the distances for And I, II, and III derived using the methodology of Rizzi et al. (2007), which 
Table 3

Summary of the ISLAndS Sample Distances and Intrinsic Properties

\begin{tabular}{|c|c|c|c|c|c|}
\hline $\begin{array}{l}\text { Galaxy } \\
\text { (1) }\end{array}$ & $\begin{array}{c}A_{V} \\
(\mathrm{mag}) \\
(2)\end{array}$ & $\begin{array}{c}(m-M)_{0} \\
(\mathrm{mag}) \\
(3)\end{array}$ & $\begin{array}{c}m_{V} \\
(\mathrm{mag}) \\
(4)\end{array}$ & $\begin{array}{c}M_{V} \\
(\mathrm{mag}) \\
(5)\end{array}$ & $\begin{array}{c}M_{\mathrm{tot}, 1 / 2} \\
\left(M_{\odot}\right) \\
(6)\end{array}$ \\
\hline And I & 0.145 & $24.51 \pm 0.08$ & $12.7 \pm 0.1$ & $-12.0 \pm 0.1$ & $3.2 \times 10^{7}$ \\
\hline And II & 0.167 & $24.17 \pm 0.07$ & $11.7 \pm 0.1$ & $-12.6 \pm 0.1$ & $3.4 \times 10^{7}$ \\
\hline And III & 0.152 & $24.38 \pm 0.06$ & $14.4 \pm 0.3$ & $-10.1 \pm 0.3$ & $2.0 \times 10^{7}$ \\
\hline And XV & 0.128 & $24.43 \pm 0.07$ & $16.0 \pm 0.3$ & $-8.4 \pm 0.3$ & $2.9 \times 10^{6}$ \\
\hline And XVI & 0.182 & $23.71 \pm 0.07$ & $16.1 \pm 0.3$ & $-7.6 \pm 0.3$ & $2.5 \times 10^{6}$ \\
\hline And XXVIII & 0.247 & $24.45 \pm 0.05$ & $15.9 \pm 0.4$ & $-8.8 \pm 0.4$ & $6.8 \times 10^{7}$ \\
\hline
\end{tabular}

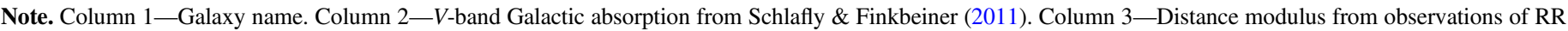

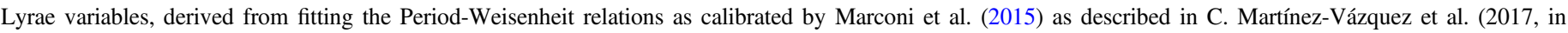

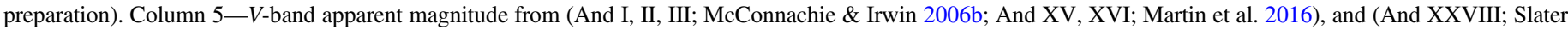

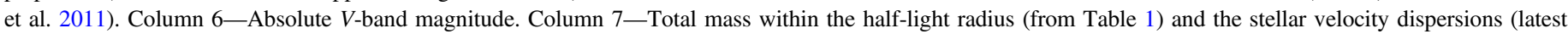
values from Table 2) and using the mass estimator from Walker et al. (2009).

is tied to the calibration of Carretta et al. (2000), differ from the PWR distances by only $0.01,0.07$, and $0.03 \mathrm{mag}$, respectively.

Conn et al. (2012) have reported TRGB distances for a large number of M31 satellite galaxies. All of our distances are larger than those derived by Conn et al. (2012). For And I and And II, due to the small associated uncertainties, the differences in distance modulus of 0.20 and 0.17 mag are significant. Conn et al. (2012) adopts the TRGB calibration of Bellazzini (2008), so it might be possible that some of this offset is due to the different calibration. For And XV and And XVI, the differences of 0.45 and 0.32 magnitudes are large, but the probability distributions for both of these galaxies have secondary peaks at larger distances which are consistent with the RR Lyrae PWR distances.

We present the distances from C. Martínez-Vázquez et al. (2017, in preparation) and some resultant intrinsic properties in Table 3. To determine the absolute magnitudes in Table 3, we used the photometry for And I, II, and III from McConnachie et al. (2004), for And XV and And XVI from Martin et al. (2016), and for And XXVIII from Slater et al. (2011). A comparison between the apparent magnitudes for the M31 satellites in McConnachie (2012) and those in Martin et al. revealed an offset for the most luminous galaxies, which could be due to some simplifying assumptions in the modeling. Thus, the early photometry of McConnachie et al. (2004) is favored for And I, II, and III. For comparison, in the final column of Table 3, we add the total mass within the half-light radius calculated from the half-light radius from Table 1 and the stellar velocity dispersions (latest values from Table 2) and using the mass estimator from Walker et al. (2009). For selfconsistency, the values of $M_{V}$ derived here are those reported in Table 1.

\subsection{Methodology}

These SFHs were derived using two parallel methods. One is known as the IAC method, consisting of IAC-star (Aparicio \& Gallart 2004), IAC-pop (Aparicio \& Hidalgo 2009), and MinnIAC (Aparicio \& Hidalgo 2009; Hidalgo et al. 2011). These codes allow simultaneous solutions for the SFH and the AMR with total uncertainties estimated from contributions from statistical, binning, distance, and reddening uncertainties. Details of the methodology can be found in Hidalgo et al. (2011). Specifically, solutions were obtained using the BaSTI
(Pietrinferni et al. 2004) stellar evolutionary libraries and the bolometric corrections of Bedin et al. (2005).

The other code is MATCH (Dolphin 2002). MATCH has been recently modified to calculate statistical uncertainties (Dolphin 2013) and systematic uncertainties associated with the choice of stellar evolution library (Dolphin 2012). For the statistical uncertainties, random uncertainties were generated using the Hybrid Monte Carlo algorithm of Duane et al. (1987). The result of this Markov Chain Monte Carlo routine is a sample of 10,000 SFHs whose density is proportional to the probability density. Upper and lower random error bars for any given value (e.g., cumulative stellar mass fraction at a particular point in time) are calculated by identifying the boundaries of the highest-density region containing $68 \%$ of the samples, with the value $68 \%$ adopted as it is the percentage of a normal distribution falling between the $\pm 1 \sigma$ bounds. The systematic uncertainties are estimated through application of shifts in temperature and luminosity, which mimic the differences between stellar evolution libraries. For the implementation of the Dolphin (2012) methodology, we used $\sigma_{\log T_{\text {eff }}}=0.013$ and $\sigma_{M_{\text {bol }}}=0.19$.

The results from the two different codes were compared and found to be in agreement within the uncertainties (when using identical stellar evolution libraries). Here we present the results based on the MATCH code. These will allow a more uniform comparison to previous studies of other galaxies in the literature (e.g., Weisz et al. 2014a).

As noted previously, the observations of And I are contaminated by the presence of the giant stellar stream. In order to account for this in reconstructing the And I SFH, a cutout region of the And I UVIS field was identified and the data were slightly smoothed in order to construct a "foreground" model (in the same way that contamination by the MW can be modeled and subtracted from lower Galactic latitude galaxies, see Dolphin 2002).

\subsection{The SFHs}

We next present a quantitative comparison of the lifetime SFHs for the six M31 dSphs in the ISLAndS sample. As discussed in the Appendix, the dominant source of systematic uncertainty in the determination of an SFH from a CMD (of a given photometric depth) is the choice of the stellar evolution library. Thus, when comparing SFHs of different galaxies, it is 


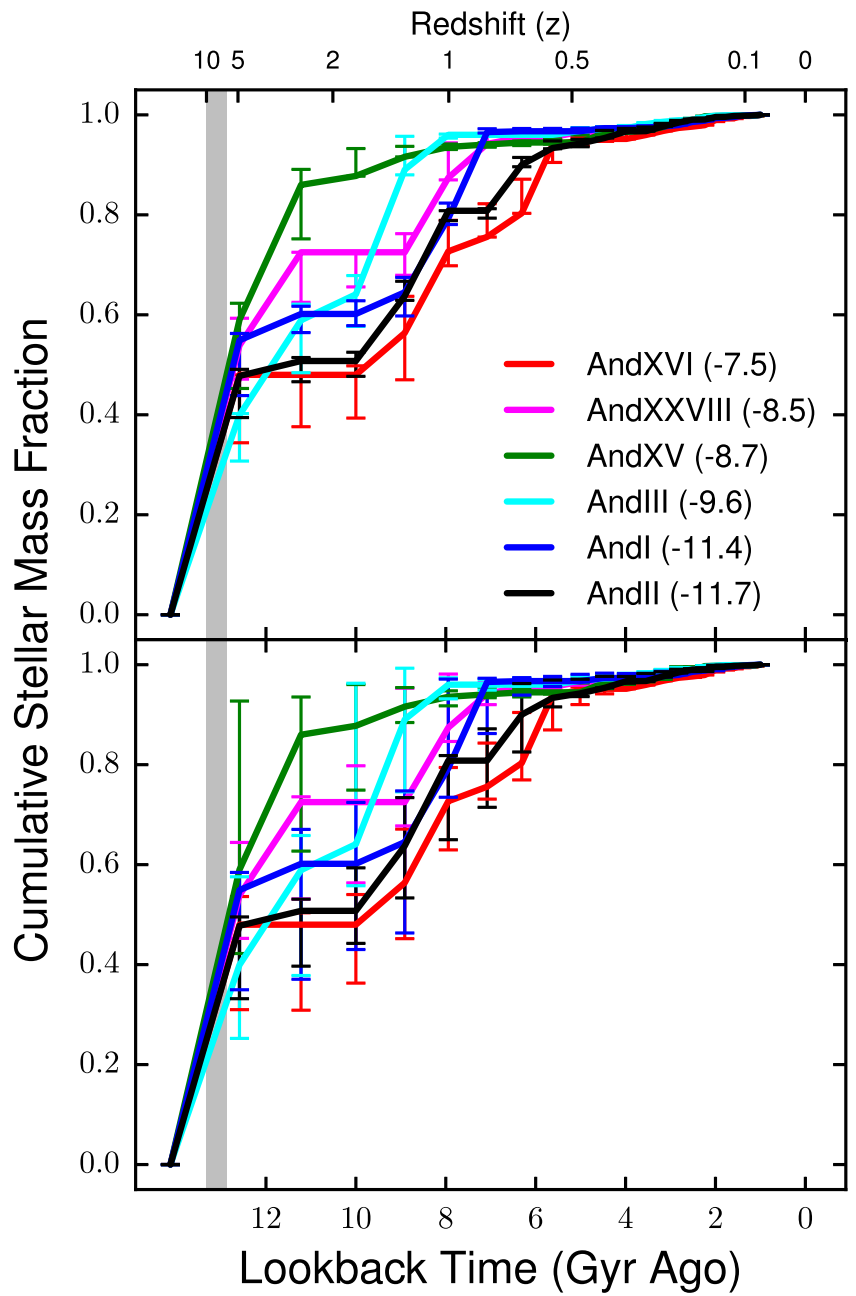

Figure 7. Comparison between the SFHs of the ISLAndS galaxies shown as cumulative stellar mass fraction. The upper panel shows only the statistical uncertainties, and the lower panel additionally accounts for estimated systematic uncertainties as discussed in Dolphin (2012, 2013). The redshift scale given on the top axis is calculated assuming a concordance $\Lambda \mathrm{CDM}$ cosmology. The shaded portion of the graph shows the era of cosmological reionization from $z \approx 10-6$. The galaxy absolute $V$ magnitudes are shown in the legend.

important to use the same library (and, ideally, to conduct the comparison multiple times with different libraries). For a first look at the SFHs of the ISLAndS sample, we use the MATCH code and the Padua (Girardi et al. 2010) stellar evolution library. We choose the Padua stellar evolution library to allow for direct comparisons with previous work (e.g., Weisz et al. 2014a).

In Figure 7, we show a comparison between the SFHs of the six ISLAndS galaxies as cumulative stellar mass fractions. In the upper panel, only the statistical uncertainties (as per Dolphin 2013) are shown. In the lower panel, the combined statistical and systematic uncertainties (as per Dolphin 2012) are presented. Because all of the ISLAndS galaxies have been observed to comparable depth, systematics in the models should have similar impacts on all of the derived galaxy SFHs. Thus, it is likely appropriate to make comparisons using the upper panel. Nonetheless, the lower panel shows the larger uncertainties encountered when trying to account for systematics. Note that even with the larger uncertainties, the six galaxies are shown to each have distinctive features in their
SFHs. The data plotted in Figure 7 are presented in Table 4. The effects of systematic uncertainties are discussed in more detail in the Appendix.

Presenting the SFHs as cumulative stellar mass fractions (as opposed to the SFRs as a function of time) is the optimal way to compare observations to theoretical models for several reasons. Variations in observed SFRs can be strongly affected by time binning and the changing time resolution as a function of lookback time. Often, it is possible to have very different impressions of a single SFH simply by changing the time binning. It is possible to match the observational time binning by reducing the resolution in the models, but using the cumulative stellar mass fraction as the diagnostic avoids this problem altogether. It is also possible to compare galaxies at any arbitrary value of the cumulative stellar mass fraction, as opposed to choosing particular values to focus on. In the comparisons that follow, we will use the cumulative stellar mass fraction as the sole diagnostic.

Note that there is one obvious failing of the cumulative stellar mass fraction as the sole diagnostic, and that is the lack of information about the absolute masses of the systems. In Figure 7, we have added the stellar luminosities to the legend for ease of comparison. It is immediately clear that the mean ages of the stellar populations do not correlate with the present stellar luminosities. And III, And XV, and And XXVIII have the faster development of their stellar contents, despite significant differences in luminosity and distance from M31. A more detailed comparison of the SFHs as a function of distance and luminosity is given in Section 7.

Figure 7 shows a variety of SFHs. However, all six SFHs have the common properties of starting their star formation early (there is significant star formation in the earliest time bin for all six galaxies), having at least $50 \%$ of their stars formed by a lookback time of $9 \mathrm{Gyr}$, and then ceasing all star formation by a lookback time of $\sim 6$ Gyr. And XV and And III show the oldest mean stellar populations of the sample, but both are clearly not consistent with a single age population and are clearly not consistent with forming all of their stars before the epoch of reionization (as indicated in Figure 7 by the shaded region). As demonstrated in Weisz et al. (2014d) and Monelli et al. (2016), And XVI has a very extended SFH, and the comparison in Figure 7 shows that it has the most extended star formation in the sample. However, conspicuously absent from Figure 7 are galaxies with very late-quenching times like the MW dSphs Carina, Fornax, and Leo I. A preliminary comparison of the ISLAndS dSph SFHs with the MW $\mathrm{dSph}$ SFHs is given in Section 7.

\section{Possible Evidence for Synchronized Quenching}

\subsection{The Quenching Time}

The definition of the quenching time for a galaxy is somewhat vague. Part of this is operational, due to the presence of BSSs. These appear as a population aged 2-3 Gyr, amounting to as much as $2 \%-3 \%$ of the stellar mass; however, there is strong evidence in favor of their interpretation as a population of merged primordial binaries with the same age as the bulk of the old stellar population. Therefore, defining the quenching time when the inferred SFR goes to zero can be misleading (see the discussion in Weisz et al. 2014b). Thus, typically a quenching time is set to a time when the cumulative star formation is approaching complete, 
Table 4

Cumulative Star Formation Histories of the ISLAndS Galaxies

\begin{tabular}{|c|c|c|c|c|c|c|}
\hline $\begin{array}{l}\text { Galaxy } \\
\text { (1) }\end{array}$ & $\begin{array}{l}\text { And I } \\
(2)\end{array}$ & $\begin{array}{l}\text { And II } \\
\text { (3) }\end{array}$ & $\begin{array}{l}\text { And III } \\
\text { (4) }\end{array}$ & $\begin{array}{l}\text { And XV } \\
(5)\end{array}$ & $\begin{array}{l}\text { And XVI } \\
(6)\end{array}$ & $\begin{array}{l}\text { And XXVIII } \\
\text { (7) }\end{array}$ \\
\hline Total Mass Formed & $6.94_{-0.01,1.1}^{+0.14,0.35}$ & $5.71_{-0.01,0.79}^{+0.01,0.64}$ & $3.49_{-0.01,0.39}^{+0.01,0.68}$ & $1.23_{-0.02,0.16}^{+0.02,0.19}$ & $0.35_{-0.01,0.06}^{+0.01,0.05}$ & $2.02_{-0.04,0.43}^{+0.02,0.17}$ \\
\hline$f_{10.1}$ & $0.55_{-0.11,0.2}^{+0.01,0.04}$ & $0.48_{-0.08,0.15}^{+0.01,0.02}$ & $0.40_{-0.09,0.15}^{+0.0,0.18}$ & $0.59_{-0.14,0.17}^{+0.03,0.34}$ & $0.48_{-0.14,0.17}^{+0.0,0.06}$ & $0.54_{-0.07,0.09}^{+0.05,0.11}$ \\
\hline$f_{10.05}$ & $0.60_{-0.04,0.23}^{+0.02,0.07}$ & $0.51_{-0.04,0.11}^{+0.01,0.02}$ & $0.59_{-0.1,0.21}^{+0.03,0.07}$ & $0.86_{-0.11,0.23}^{+0.03,0.08}$ & $0.48_{-0.1,0.17}^{+0.0,0.02}$ & $0.73_{-0.1,0.19}^{+0.0,0.01}$ \\
\hline$f_{10.0}$ & $0.60_{-0.02,0.17}^{+0.03,0.12}$ & $0.51_{-0.03,0.07}^{+0.02,0.09}$ & $0.64_{-0.06,0.08}^{+0.04,0.32}$ & $0.88_{-0.0,0.13}^{+0.05,0.08}$ & $0.48_{-0.09,0.12}^{+0.02,0.06}$ & $0.73_{-0.07,0.16}^{+0.0,0.07}$ \\
\hline$f_{9.95}$ & $0.65_{-0.05,0.18}^{+0.03,0.1}$ & $0.64_{-0.01,0.1}^{+0.03,0.1}$ & $0.89_{-0.01,0.14}^{+0.07,0.1}$ & $0.92_{-0.0,0.03}^{+0.02,0.04}$ & $0.56_{-0.09,0.11}^{+0.07,0.11}$ & $0.73_{-0.05,0.05}^{+0.04,0.23}$ \\
\hline$f_{9.9}$ & $0.79_{-0.01,0.06}^{+0.03,0.18}$ & $0.81_{-0.02,0.16}^{+0.0,0.01}$ & $0.96_{-0.01,0.03}^{+0.0,0.01}$ & $0.94_{-0.01,0.02}^{+0.01,0.01}$ & $0.73_{-0.03,0.1}^{+0.06,0.07}$ & $0.87_{-0.0,0.03}^{+0.07,0.11}$ \\
\hline$f_{9.85}$ & $0.97_{-0.0,0.1}^{+0.01,0.01}$ & $0.81_{-0.01,0.09}^{+0.0,0.06}$ & $0.96_{-0.0,0.01}^{+0.0,0.01}$ & $0.94_{-0.01,0.01}^{+0.0,0.01}$ & $0.76_{-0.0,0.02}^{+0.07,0.09}$ & $0.94_{-0.0,0.02}^{+0.02,0.02}$ \\
\hline$f_{9.8}$ & $0.97_{-0.0,0.03}^{+0.01,0.01}$ & $0.90_{-0.0,0.07}^{+0.01,0.06}$ & $0.96_{-0.0,0.0}^{+0.0,0.01}$ & $0.94_{-0.01,0.01}^{+0.0,0.01}$ & $0.80_{-0.0,0.03}^{+0.07,0.1}$ & $0.96_{-0.0,0.02}^{+0.01,0.01}$ \\
\hline$f_{9.75}$ & $0.97_{-0.0,0.01}^{+0.01,0.01}$ & $0.93_{-0.0,0.02}^{+0.01,0.04}$ & $0.96_{-0.0,0.0}^{+0.01,0.01}$ & $0.94_{-0.0,0.0}^{+0.01,0.01}$ & $0.94_{-0.03,0.07}^{+0.01,0.01}$ & $0.96_{-0.0,0.0}^{+0.01,0.01}$ \\
\hline$f_{9.7}$ & $0.97_{-0.0,0.0}^{+0.01,0.01}$ & $0.94_{-0.01,0.01}^{+0.01,0.03}$ & $0.97_{-0.0,0.0}^{+0.01,0.01}$ & $0.94_{-0.0,0.0}^{+0.02,0.02}$ & $0.95_{-0.01,0.03}^{+0.01,0.01}$ & $0.97_{-0.0,0.01}^{+0.0,0.01}$ \\
\hline$f_{9.65}$ & $0.97_{-0.0,0.0}^{+0.0,0.01}$ & $0.95_{-0.0,0.0}^{+0.02,0.02}$ & $0.97_{-0.0,0.0}^{+0.0,0.01}$ & $0.96_{-0.0,0.01}^{+0.01,0.01}$ & $0.95_{-0.0,0.01}^{+0.01,0.01}$ & $0.97_{-0.0,0.0}^{+0.01,0.01}$ \\
\hline$f_{9.6}$ & $0.97_{-0.0,0.0}^{+0.0,0.01}$ & $0.97_{-0.0,0.0}^{+0.01,0.01}$ & $0.97_{-0.0,0.0}^{+0.01,0.01}$ & $0.97_{-0.0,0.01}^{+0.01,0.01}$ & $0.95_{-0.0,0.0}^{+0.01,0.02}$ & $0.97_{-0.0,0.0}^{+0.0,0.0}$ \\
\hline$f_{9.55}$ & $0.97_{-0.0,0.0}^{+0.0,0.01}$ & $0.97_{-0.0,0.0}^{+0.01,0.02}$ & $0.98_{-0.0,0.0}^{+0.0,0.0}$ & $\begin{array}{c}0.97_{-0.0,0.0}^{+0.01,0.01} \\
\end{array}$ & $0.96_{-0.0,0.0}^{+0.01,0.02}$ & $0.97_{-0.0,0.0}^{+0.01,0.01}$ \\
\hline$f_{9.5}$ & $0.98_{-0.0,0.0}^{+0.0,0.01}$ & $0.98_{-0.0,0.0}^{+0.01,0.01}$ & $0.99_{-0.0,0.0}^{+0.0,0.0}$ & $0.98_{-0.0,0.01}^{+0.01,0.01}$ & $0.97_{-0.0,0.0}^{+0.01,0.01}$ & $0.98_{-0.0,0.0}^{+0.01,0.01}$ \\
\hline$f_{9.45}$ & $0.98_{-0.0,0.0}^{+0.01,0.01}$ & $0.98_{-0.0,0.0}^{+0.0,0.01}$ & $0.99_{-0.0,0.0}^{+0.0,0.01}$ & $0.99_{-0.0,0.0}^{+0.01,0.01}$ & $0.97_{-0.0,0.0}^{+0.01,0.01}$ & $0.98_{-0.0,0.0}^{+0.0,0.01}$ \\
\hline$f_{9.4}$ & $0.99_{-0.0,0.0}^{+0.0,0.0}$ & $0.99_{-0.0,0.0}^{+0.0,0.0}$ & $0.99_{-0.0,0.0}^{+0.0,0.0}$ & $0.99_{-0.0,0.0}^{+0.0,0.01}$ & $0.98_{-0.0,0.0}^{+0.01,0.01}$ & $0.99_{-0.0,0.0}^{+0.0,0.0}$ \\
\hline$f_{9.35}$ & $0.99_{-0.0,0.0}^{+0.0,0.0}$ & $0.99_{-0.0,0.0}^{+0.0,0.0}$ & $1.00_{-0.0,0.0}^{+0.0,0.0}$ & $1.00_{-0.0,0.0}^{+0.0,0.0}$ & $0.98_{-0.0,0.0}^{+0.01,0.01}$ & $0.99_{-0.0,0.0}^{+0.0,0.0}$ \\
\hline$f_{9.3}$ & $0.99_{-0.0,0.01}^{+0.0,0.0}$ & $1.00_{-0.0,0.0}^{+0.0,0.0}$ & $1.00_{-0.0,0.0}^{+0.0,0.0}$ & $1.00_{-0.0,0.0}^{+0.0,0.0}$ & $0.99_{-0.0,0.0}^{+0.0,0.0}$ & $1.00_{-0.0,0.0}^{+0.0,0.0}$ \\
\hline$f_{9.25}$ & $0.99_{-0.0,0.0}^{+0.0,0.0}$ & $1.00_{-0.0,0.0}^{+0.0,0.0}$ & $1.00_{-0.0,0.0}^{+0.0,0.0}$ & $1.00_{-0.0,0.0}^{+0.0,0.0}$ & $0.99_{-0.0,0.0}^{+0.0,0.0}$ & $1.00_{-0.0,0.0}^{+0.0,0.0}$ \\
\hline$f_{9.2}$ & $1.00_{-0.0,0.0}^{+0.0,0.0}$ & $1.00_{-0.0,0.0}^{+0.0,0.0}$ & $1.00_{-0.0,0.0}^{+0.0,0.0}$ & $1.00_{-0.0,0.0}^{+0.0,0.0}$ & $0.99_{-0.0,0.0}^{+0.0,0.0}$ & $1.00_{-0.0,0.0}^{+0.0,0.0}$ \\
\hline$f_{9.15}$ & $1.00_{-0.0,0.0}^{+0.0,0.0}$ & $1.00_{-0.0,0.0}^{+0.0,0.0}$ & $1.00_{-0.0,0.0}^{+0.0,0.0}$ & $1.00_{-0.0,0.0}^{+0.0,0.0}$ & $1.00_{-0.0,0.0}^{+0.0,0.0}$ & $1.00_{-0.0,0.0}^{+0.0,0.0}$ \\
\hline$f_{9.1}$ & $1.00_{-0.0,0.0}^{+0.0,0.0}$ & $1.00_{-0.0,0.0}^{+0.0,0.0}$ & $1.00_{-0.0,0.0}^{+0.0,0.0}$ & $1.00_{-0.0,0.0}^{+0.0,0.0}$ & $1.00_{-0.0,0.0}^{+0.0,0.0}$ & $1.00_{-0.0,0.0}^{+0.0,0.0}$ \\
\hline$f_{9.05}$ & $1.00_{-0.0,0.0}^{+0.0,0.0}$ & $1.00_{-0.0,0.0}^{+0.0,0.0}$ & $1.00_{-0.0,0.0}^{+0.0,0.0}$ & $1.00_{-0.0,0.0}^{+0.0,0.0}$ & $1.00_{-0.0,0.0}^{+0.0,0.0}$ & $1.00_{-0.0,0.0}^{+0.0,0.0}$ \\
\hline$f_{9.0}$ & $1.00_{-0.0,0.0}^{+0.0,0.0}$ & $1.00_{-0.0,0.0}^{+0.0,0.0}$ & $1.00_{-0.0,0.0}^{+0.0,0.0}$ & $1.00_{-0.0,0.0}^{+0.0,0.0}$ & $1.00_{-0.0,0.0}^{+0.0,0.0}$ & $1.00_{-0.0,0.0}^{+0.0,0.0}$ \\
\hline
\end{tabular}

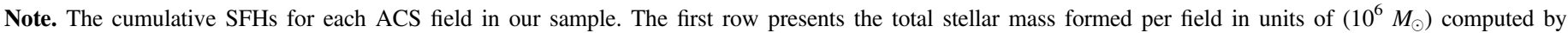

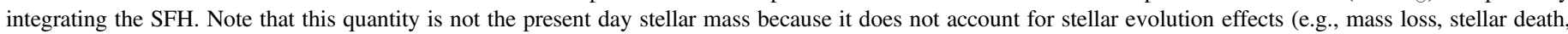

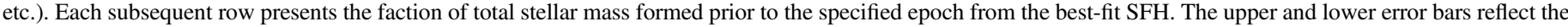

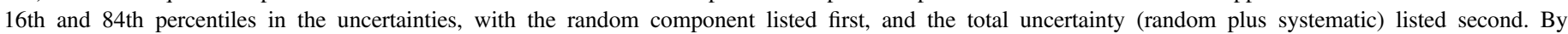
construction, the cumulative SFHs are 0 at $\log (t)=10.15$ and 1 at $\log (t)=9.0$.

but something less than $95 \%$ of the total mass of stars has formed. For the purpose of identifying an "event" that caused the quenching, it might make sense to allow for star formation that follows the quenching. For example, Ricotti \& Gnedin (2005) and Bovill \& Ricotti (2011) promote the use of the age of $70 \%$ of stars formed as a criterion for distinguishing true fossil of reionization from other galaxies. This $70 \%$ criterion fails as a quenching identification in galaxies with extended star formation. For our purposes of searching for synchronized quenching, we will adopt an age when $90 \%$ of the stars have formed as a measure of the quenching time $\left(\tau_{q}\right)$. The $90 \%$ serves as a very robust measurement of the quenching time as it is not affected by the presence of BSSs.

\subsection{Synchronized Quenching?}

Weisz et al. (2014d) noted that the SFHs of And II and And XVI showed nearly identical quenching at a lookback time of approximately six Gyr. Weisz et al. (2014a) additionally noted that shallow CMDs of And I, And III, And XI, and And XII also indicated star formation extending to intermediate ages, but the shallow depths of these CMDs precluded strong inferences. This possibility became even more intriguing with the addition of SFHs based on deep HST CMDs of NGC 147 and NGC 185 by Geha et al. (2015). The SFH of NGC 147 is consistent with a quenching time of 6 Gyr. Although the SFH of NGC 185 shows it to be almost entirely old, the uncertainties allow for continuing star formation up until a lookback time of $\sim 7$ Gyr. However, as noted in the Introduction, because the observed fields in NGC 147 and NGC 185 are outside of the half-light radius, the interpretation and comparison of their SFHs is a bit problematic.

The addition of the new deep CMDs allow us to test for the possibility of synchronized quenching in the M31 satellites. Indeed, the new SFHs give no support for synchronized quenching. Specifically, And XV appears to have formed $90 \%$ of its stars much earlier at $\sim 9$ Gyr, And III and And XXVIII at a lookback time of $\sim 8 \mathrm{Gyr}$, and And I at a lookback time of $\sim 7.5$ Gyr.

Weisz et al. (2014d) offered two other explanations for the synchronized quenching in And II and And XVI: (1) that both galaxies may have similar halo masses with similar gas retention timescales (implying significant differences in stellar mass at a fixed halo mass, see, e.g., Boylan-Kolchin et al. 2011; Garrison-Kimmel et al. 2014), or (2) coincidental near passes with M31 transforming previously gas-rich dwarfs into gaspoor dSphs via "tidal stirring" (Mayer et al. 2001a). Proper 
motions of these galaxies will be required to define their orbits in order to distinguish between these options.

\subsection{Quenching by Reionization?}

Our understanding of the effect of reionization on dwarf galaxies is primarily driven by studies of MW companion galaxies with photometry that reaches below the oMSTO. Thus, it is important to know if the MW satellites are representative in this context or if the assembly of the MW and its satellite system is affecting our interpretations. Our new imaging to below the oMSTO of a sample of M31 dwarfs allows us to explore this question for the first time. The full details of the earliest SFHs will be deferred to a later study where the inherent time resolution will be carefully modeled (e.g., Monelli et al. 2010a, 2010b; Hidalgo et al. 2011, 2013), and comparisons will be made to the most recent galaxy evolution models. Nonetheless, we present here a brief overview of the potential impact that these observations will have on the study of the effects of reionization on the SFHs of dwarf galaxies.

Weisz et al. (2014b) presented a review of the theoretical predictions and observational tests connecting the cosmic UV background from reionization with the quenching of star formation in dwarf galaxies. We provide a very brief summary here. Quenching of star formation in dSphs by the cosmic UV background has been investigated multiple times (e.g., Efstathiou 1992; Bullock et al. 2000; Benson et al. 2002, 2003; Somerville 2002; Kravtsov et al. 2004; Okamoto et al. 2008). With time, the expected imprint of the cosmic UV background on the SFHs of dwarf galaxies has evolved. Many early studies assumed that sufficiently low-mass galaxies would have their star formation permanently quenched by reionization on a very short timescale. Thus, it was tempting to connect the lack of current star formation in the dSph galaxies with complete quenching by reionization. Grebel \& Gallagher (2004) pointed out that the observed SFHs of the Local Group dwarfs did not support this simple picture.

Later models allowed for some star formation after reionization (e.g., Ricotti \& Gnedin 2005; Gnedin \& Kravtsov 2006; Bovill \& Ricotti 2009; Sawala et al. 2010), which reduced the tension somewhat between the models and the observations of Local Group dSph galaxies. However, recent, high-resolution simulations of dwarf galaxies show that the impact of reionization can be much more subtle than originally imagined. Supporting the original suggestion by Susa \& Umemura (2004) and Dijkstra et al. (2004) that radiative transfer effects are important, recent work indicates that the cosmic UV background may suppress infall of fresh gas, but is not likely to boil away cold gas already present (e.g., Oñorbe et al. 2015). This means that searching for the signature of reionization in the SFHs of dwarf galaxies is much more complicated than originally envisioned. The simulations of Wheeler et al. (2015) provide a dividing line of the peak virial mass, $M_{\text {peak }},=5 \times 10^{9} M_{\odot}$, below which their model galaxies show star formation to be entirely quenched, but only by $z \sim 2$ (a look back time of $\sim 10 \mathrm{Gyr}$ ).

It is clear that the SFHs of all six galaxies are extended and inconsistent with a single age stellar population. How does this compare with expectations? In the models of Wheeler et al. (2015), $M_{\text {peak }}=5 \times 10^{9} M_{\odot}$ corresponds to present day stellar masses of $\sim 3 \times 10^{5} M_{\odot}$. In this regard, assuming a $V$-band mass-to-light ratio of 1 , And XVI is below this dividing line,
And III, And XV, and And XXVIII are close to this dividing line, and And I and And II are well above this division. Table 1 presents the circular velocities measured at the half-light radii for the six ISLAndS galaxies as reported in Collins et al. (2014) and Tollerud et al. (2014). These range from $\sim 6 \mathrm{~km} \mathrm{~s}^{-1}$ (And XV) to $\sim 18 \mathrm{~km} \mathrm{~s}^{-1}$ (And I). The values of $M_{\mathrm{tot}, 1 / 2}$ presented in Table 3 from the Walker et al. (2009) mass estimator (which is consistent within the observational uncertainties with that of Wolf et al. 2010) range from $\sim 3 \times 10^{6} M_{\odot}$ (And XV and And XVI) to $\sim 3 \times 10^{7} M_{\odot}$ (And I and And II). Thus, from stellar velocity dispersions, And XV and And XVI are right at the limit of where galaxies are expected to have their star formation quenched by reionization. The SFH of And XV is possibly consistent with the models of Wheeler et al. (2015), showing the star formation to be almost entirely quenched at a lookback time of 9 Gyr. However, And XVI shows the most extended SFH of the sample. As pointed out by Weisz et al. (2014d) and Monelli et al. (2016), the SFH of And XVI, with its large intermediate-age population and continuous star formation, is inconsistent with complete quenching by reionization. The lack of correlation of quenching time with measures of the halo mass (e.g., the values of $V_{c, 1 / 2}$ listed in Table 1), puts strong constraints on the potential role of quenching by reionization in this galaxy sample (in agreement with results from similar observations of the MW dSph satellites).

Much of the literature discussion focuses on reionization quenching, which completely terminates star formation at the reionization epoch or shortly thereafter. However, we would like to also look for possibly less catastrophic impacts of reionization, where star formation is temporarily slowed or paused and resumes at a later time. One thing that is interesting about the SFH of And XVI is that the SFR decreases immediately after the post-reionization period. We may be seeing evidence of the impact of the ultraviolet background on the star formation in And XVI, but the result is not a complete quenching. And I and And II also show inflections in their cumulative stellar mass fractions indicating a slowdown in the formation of stars immediately post-reionization. This is certainly interesting in the context of any kind of link between reionization and suppression of SFR. It is also interesting that the SFHs of And III, And XV, and And XXVIII show little evidence of a decrease in SFR in the immediate postreionization period (and demonstrates that the slowdown seen in the other galaxies is not a systematic effect from the CMD modeling). We will focus on these interesting features in our future detailed studies of the earliest SFHs, with special focus on the robust characteristics that are independent of the stellar evolution library.

\section{Is There a Thin Plane/Non-thin Plane Dichotomy?}

Ibata et al. (2013) discovered that about half of the satellites of M31 reside in a planar subgroup. This vast, thin structure is at least $400 \mathrm{kpc}$ in diameter, but less than $14 \mathrm{kpc}$ in width. The member satellites rotate about M31 in the same sense. The reality and probability of this plane has been discussed at length, especially with regard to whether such a structure is consistent with galaxy formation in a $\Lambda$ CDM cosmology (see, e.g., Hammer et al. 2013; Bahl \& Baumgardt 2014; Ibata et al. 2014; Pawlowski et al. 2014; Buck et al. 2015; Gillet et al. 2015; Libeskind et al. 2015; Phillips et al. 2015). 
Collins et al. (2015) have investigated the possibility that inplane galaxies and off-plane galaxies may have formed and evolved differently. The different evolutionary paths could result in different chemistries, dynamics, and SFHs. From a comparison of the relationships between the velocity dispersions, masses, half-light radii, luminosities, and metallicities available for all of the M31 dSphs, Collins et al. (2015) found the in- and off-plane Andromeda dwarf galaxies to be indistinguishable from one another.

One characteristic that was not available to Collins et al. (2015) was the lifetime SFHs of the M31 satellites. For the sample here, three of the galaxies (And I, And III, and And XVI) are in the plane and three of the galaxies (And II, And XV, and And XXVIII) are not (see Figure 2). Comparing the SFHs, we also find no evidence for differences between the in- and off-plane galaxies. Specifically, And II and And XVI are the two galaxies with the most extended SFHs, yet one is off-plane and the other is in-plane. And III, And XV, and And XXVIII are the three galaxies with the earliest truncation times, and one is in-plane and the other two are off-plane. It would appear that the physical process leading to the distinction of in-plane and off-plane galaxies does not leave an imprint on any of the intrinsic properties of the galaxies.

\section{Comparing The Lifetime SFHs of the M31 and MW Satellites}

We reserve a detailed examination of the earliest SFHs of the ISLAndS galaxies for a follow-up study. The SFHs of the first few gigayears entails precise modeling of the intrinsic time resolution of the observations (see discussions in Hidalgo et al. 2011; Aparicio et al. 2016) and we are also planning to use the latest updates for the MESA (Dotter 2016) and BASTI stellar evolution libraries. Nonetheless, interesting comparisons of the very robust late-time features of the SFHs can be made at this time with great confidence.

Weisz et al. (2014d) provided an initial glimpse at comparing SFHs of M31 and MW dSphs by comparing the SFHs of And II and And XVI to MW dSphs with comparable luminosities and at comparable host distances. As the number of galaxies with deep CMDs allowing for secure SFHs increases, it is desirable to produce a diagnostic diagram in which the comparison is simplified. Two key characteristics of an SFH are the time required to produce $50 \%\left(\tau_{50}\right)$ of the total star formation and the quenching time $\left(\tau_{q}\right)$. The time for the production of $50 \%$ of the total stars can be measured robustly and gives a first order characterization separating galaxies that form quickly from those with a more steady build up of stars. As discussed earlier, the time associated with $90 \%$ of the total star formation gives a good approximation for the quenching time, which is free from contamination by the presence of BSSs.

We can use these two characteristics to construct three diagnostic diagrams to compare the MW and M31 dSph SFHs: (1) a comparison of the times when $50 \%$ and $90 \%$ of the stars are formed as a function of current distance to the host galaxy; (2) a comparison of the times when $50 \%$ and $90 \%$ of the stars are formed as a function of the luminosity of the galaxy; and (3) a direct comparison of the times when $50 \%$ and $90 \%$ of the stars are formed. Here we use the SFHs for the MW dSphs taken from Weisz et al. (2014a) to compare to the ISLAndS $\mathrm{dSphs}$ studied here. There are two advantages to this MW dSphs comparison set. First, both studies have used the Padova

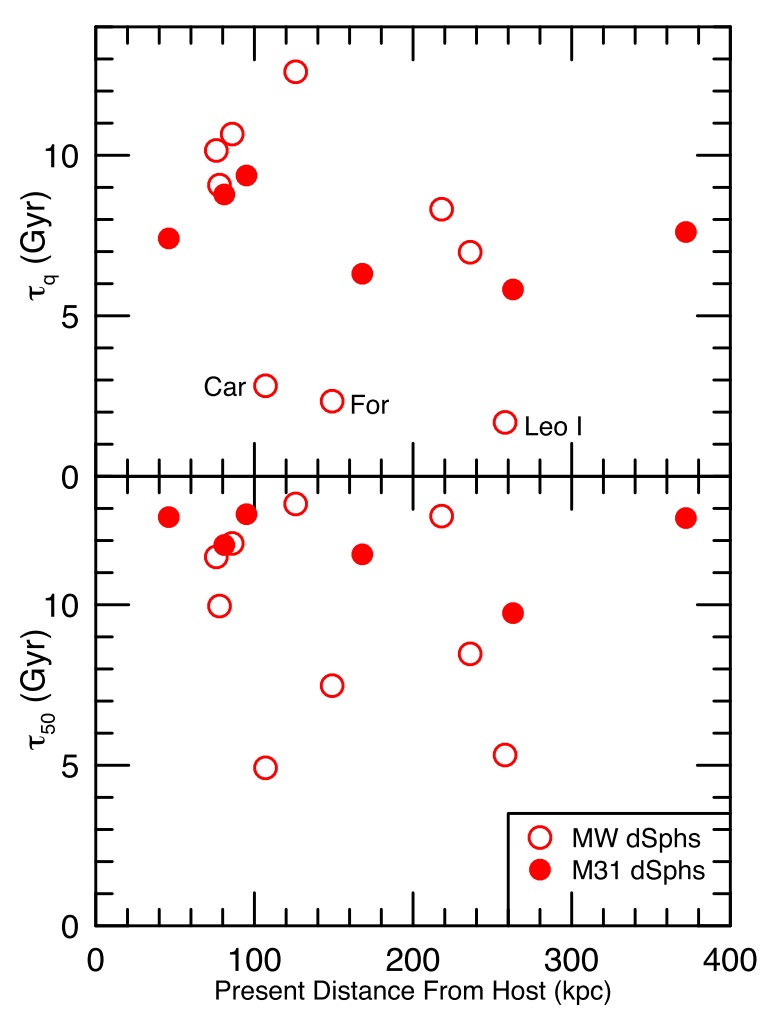

Figure 8. Comparison of the lookback times for 50\% $\left(\tau_{50}\right.$, lower panel) and $90 \%$ ( $\tau_{q}$, upper panel) of the stars to form as a function of distance from the MW for the MW dSphs taken from Weisz et al. (2014a) and the ISLAndS dSphs studied here. Note that there are no M31 dSphs with very late ( $\leqslant 5$ Gyr) quenching times. The three MW dSphs with very late-quenching times (Carina, Fornax, and Leo I) are labeled. Note also that there is only a weak trend in the importance of intermediate-age populations as a function of present distance from the host galaxy for both samples.

stellar evolution library (Girardi et al. 2010), so the systematic offsets resulting from choice of library, while not as important for the later times concerned here, are minimized. Second, the SFHs are calculated in an identical, uniform way, so that direct comparisons are valid. In order to provide a fair comparison, we restrict the MW comparison sample to a range in an absolute luminosity of $-14 \leqslant M_{V} \leqslant-6$. This range encompasses the range from the ISLAndS sample.

One disadvantage of the Weisz et al. (2014a) sample is the small $H S T$ field of view (and correspondingly relatively small number of stars measured) for the closest MW companions. In this regard, ground-based observations extending below the oMSTO (e.g., Okamoto et al. 2008, 2012) can be competitive for providing accurate SFHs. As part of the ISLAndS project, we are re-analyzing a large collection of ground-based observations of MW companion galaxies to produce a single, uniform analysis. For this reason, we consider the following comparisons preliminary, and will re-visit the exercise with well-defined uncertainties for all MW companion galaxies at a later date. Nonetheless, we feel that the patterns revealed in this study are quite robust, even if the individual data points may experience small shifts in the final analysis. Note also that these comparisons are all in the regime of small number statistics. A single addition or subtraction of a galaxy can make a significant impact on the impression of these comparisons. Thus, it is best to focus on broad trends. 


\subsection{Trends with Host Distance}

Figure 8 shows the comparison of the lookback times for $90 \%$ ( $\tau_{q}$; upper panel) and $50 \%\left(\tau_{50}\right.$; lower panel) of the stars to form as a function of distance from the host galaxy for the MW dSphs and the ISLAndS dSphs studied here. Starting with the top panel, qualitatively, there are a few distinctions that can be made between the distributions in $\tau_{q}$ versus distance. The MW $\mathrm{dSphs}$ clearly divide into six early-quenching and three latequenching galaxies $(\leqslant 5 \mathrm{Gyr})$. The three $\mathrm{MW} \mathrm{dSphs}$ galaxies with very late-quenching times are Carina, Fornax, and Leo I, and these are labeled in Figure 8. Interestingly, the ISLAndS sample shows a complete lack of very late-quenching times. Comparing the mean quenching times, the M31 dSphs have a mean of 7.6 $\pm 1.4 \mathrm{Gyr}$ compared to $7.2 \pm 4.0 \mathrm{Gyr}$ for the MW $\mathrm{dSphs}$ (where the uncertainty represents the sample standard deviation). If we sub-divide the MW sample, the mean for the early-quenching galaxies is $9.6 \pm 2.0 \mathrm{Gyr}$ compared to a mean for the late-quenching galaxies of $2.3 \pm 0.6 \mathrm{Gyr}$. So, although the M31 and MW samples have very similar mean values of $\tau_{q}$, the early-quenching MW dSphs quench, on average, 2.0 Gyr before the M31 dSphs, and the late-quenching MW dSphs quench, on average, $5.3 \mathrm{Gyr}$ after the M31 dSphs. This strong dichotomy between the two MW sub-samples, which is not seen at all in the M31 sample is quite striking. Note the possible exception that, at a higher luminosity, NGC 147 does have a late-quenching time (Geha et al. 2015).

Concerning the trend of quenching times with distance from host, it is logical to restrict the comparison to only the earlyquenching galaxies. The MW satellites are not spread out evenly in distance, with four galaxies at distances between 60 and $140 \mathrm{kpc}$ and two between 200 and $240 \mathrm{kpc}$. The average quenching time for the inner MW dSphs is $10.6 \mathrm{Gyr}$, compared to the average quenching time for the outer MW dSphs of 7.7 Gyr. So, there is evidence of a radial gradient in quenching times for the early-quenching MW dSphs. The M31 sample shows a small dispersion in quenching times, but there is evidence of a slight gradient. The average quenching time for the inner three M31 dSphs, which are between 40 and $100 \mathrm{kpc}$ is $8.5 \mathrm{Gyr}$, compared to $6.6 \mathrm{Gyr}$ for the outer M31 dSphs between 150 and $380 \mathrm{kpc}$. So, there is a measurable gradient, but note that the outermost M31 dSph, And XXVIII, with a quenching time of $7.6 \mathrm{Gyr}$, is consistent with the range for the inner M31 dSphs.

In the lower panel of Figure 8, showing $\tau_{50}$ as a function of distance from host, there is little evidence for a dichotomy between the early- and late- quenching MW dSphs. There is also no evidence for a trend with distance. The average value for $\tau_{50}$ in the MW dSph sample is $9.5 \pm 3.1 \mathrm{Gyr}$. The ISLAndS sample, with a mean value of $\tau_{50}$ of $11.9 \pm 1.2 \mathrm{Gyr}$, shows a mild trend for decreasing $\tau_{50}$ with increasing present distance. Quantitatively, the average value of $\tau_{50}$ for the inner three galaxies is $12.5 \mathrm{Gyr}$ compared to $11.3 \mathrm{Gyr}$ for the outer three M31 dSphs (and again, the outermost galaxy, And XXVIII, has a value consistent with the inner sub-sample). All of the galaxies in the ISLAndS sample have formed 50\% of their stars before a lookback time of $9 \mathrm{Gyr}$, compared to only five of the nine MW dSphs.

The trend for the stellar content of faint Local Group dwarfs to correlate with distance from the Galaxy identified by van den Bergh (1994b), is not very apparent when restricted to the $\mathrm{dSph}$ population in the present luminosity range. The interpretation of this trend is complicated by the fact that these galaxies can be on non-circular orbits, and their present host distances may not be representative of their locations at the times that we are comparing them. As pointed out by Bullock \& Johnston (2005) the average infall times for surviving satellite systems have a median of $\sim 5 \mathrm{Gyr}$ in the past, so the typical surviving satellite would have been outside of the gravitational influence of the host galaxy when the bulk of its star formation occurred. Detailed modeling by Wetzel et al. (2015a) has confirmed this picture and further highlighted the "pre-processing" by other gravitational interactions before falling into the present day host. Benítez-Llambay et al. (2013) have also pointed to the potential processing of dwarf galaxies by "cosmic web stripping," which adds further complications in understanding the histories of dwarfs galaxies. The broad trend observed by van den Bergh (1994b) comparing diverse morphological types should not necessarily be expected to be obvious in a sample restricted to only dSph galaxies with present day distances. Clearly, adding orbital information is highly desirable to allow better formed questions to be investigated.

\subsection{Trends with Galaxy Luminosity}

It is possible that Figure 8 could be biased by not comparing similar luminosity MW dSphs with M31 dSphs. Although the range in absolute luminosity was restricted, that does not necessarily mean there is no bias in comparing the two samples. To test for such a possible sample bias, in Figure 9, we plot the characteristic times versus the galaxy $V$-band luminosities. The comparison for quenching times is shown in the top panel. In the top panel, we have added a dotted line corresponding to a redshift of 5 when reionization has been fully completed (Becker et al. 2015). Galaxies that were completely quenched by reionization would lie above this line. Only the very lowest luminosity galaxy in the MW sample (Hercules) is consistent with complete quenching by reionization.

Overall, the top panel of Figure 9 shows a stronger trend in the MW dSphs with luminosity than we saw when plotted as a function of present distance. The more luminous MW dSphs tend, on average, to have later quenching times. Quantitatively, the MW dSphs less luminous than -9 have an average $\tau_{q}$ of 10.0 Gyr, while those more luminous than -9.5 have an average $\tau_{q}$ of $5.4 \mathrm{Gyr}$. The baseline in luminosity for the ISLAndS sample is slightly smaller, but there is little evidence of a trend of quenching time with luminosity in the ISLAndS sample. Quantitatively, the average value of $\tau_{q}$ for the more luminous three galaxies in the ISLAndS sample $(7.5 \mathrm{Gyr})$ is nearly identical to the average for the three less luminous galaxies ( $7.6 \mathrm{Gyr})$. Indeed, the M31 dSphs appear to be much more homogeneous set in this regard. From Figure 9, it is clear that the lack of very late-quenching times in the ISLAndS sample is not due to the luminosities of the sample galaxies.

The bottom panel of Figure 9 essentially reflects the trends seen in the top panel. The $\tau_{50}$ values for the MW dSphs show a trend with luminosity in the sense of later star formation for higher luminosities. Quantitatively, the average value of $\tau_{50}$ for the four least luminous M31 dSphs is $11.8 \mathrm{Gyr}$ compared to a value of $8.3 \mathrm{Gyr}$ the four most luminous. Again the ISLAndS sample gives no evidence for a trend with average $\tau_{50}$ values of 12.1 Gyr for the three most luminous galaxies compared 


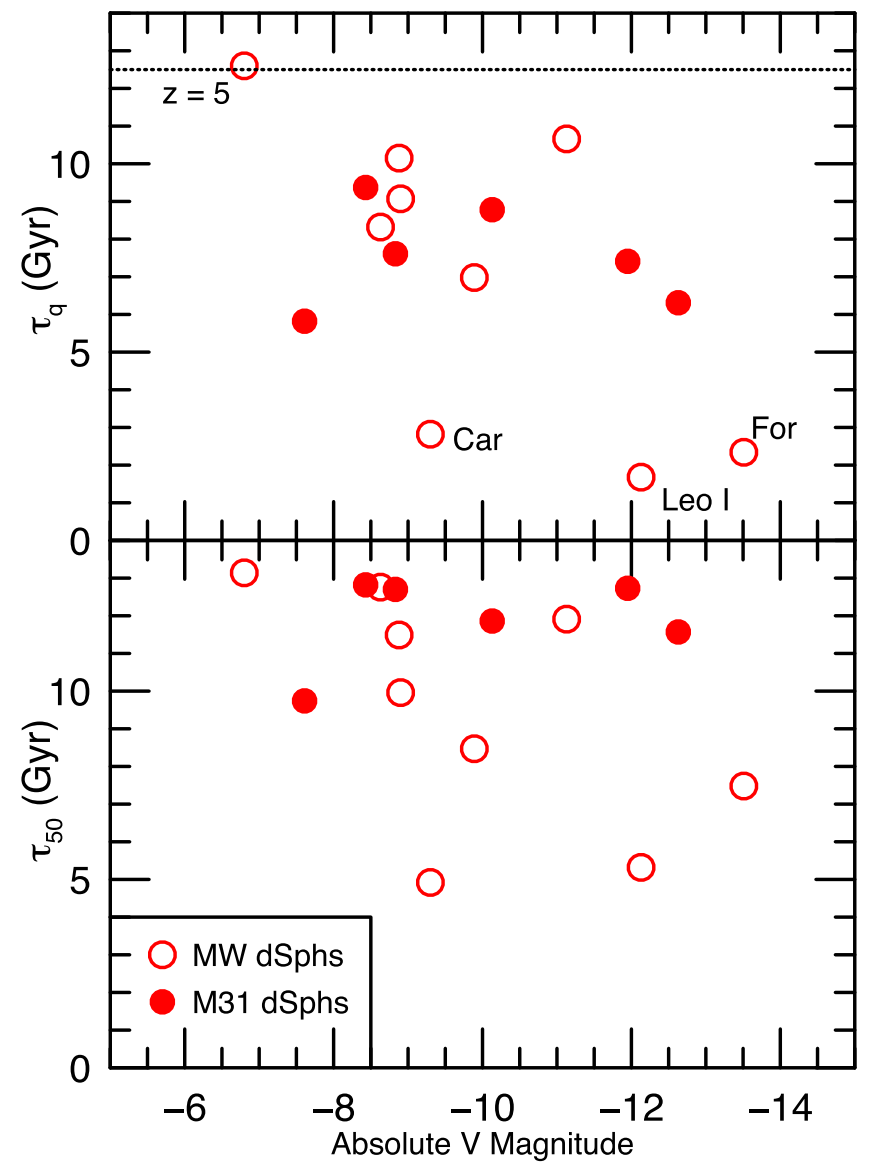

Figure 9. Comparison of the lookback times for $90 \%\left(\tau_{q}\right.$; upper panel) and $50 \%\left(\tau_{50}\right.$; lower panel) of the stars to form as a function of galaxy luminosity for the MW dSphs taken from Weisz et al. (2014a) and the ISLAndS dSphs studied here. The dotted line in the upper panel corresponds to a redshift of 5 when reionization has been fully completed (Becker et al. 2015). Galaxies that were completely quenched by reionization would lie above this line. Note that there is a trend for later quenching with increasing luminosity for both dSph populations. Note also the difference between the MW and M31 populations in their lookback times for $50 \%$ of the the stars to form.

to $11.8 \mathrm{Gyr}$ for the three lower luminosity galaxies. How much of the two trends for the MW dSphs in Figure 9 is due to the presence of the three late-quenching galaxies is difficult to say. If they are removed from the comparison, then the five remaining galaxies still show a trend, albeit very much weaker, where the impression of a trend can be altered by the presence/ absence of a single galaxy. In this regard, the absence of a trend in luminosity in the ISLAndS sample cannot be definitively seen as different from the MW sample. The main difference remains that the ISLAndS sample has no very-late-quenching galaxies, while these are common in the MW satellites of the same luminosity.

Comparing $\tau_{q}$ values (top panel) with the $\tau_{50}$ values (lower panel), there is another potentially interesting difference between the two samples. The M31 satellites have, on average, produced $50 \%$ of their stars much faster that the MW satellite. On the other hand, the M31 satellites have quenching times comparable to those of the MW satellites. Taken at face value, then this could mean that the M31 satellites experienced a much stronger early impact than the MW dwarfs. That is, star formation was greatly suppressed in the M31 satellites and then only returned back to comparable

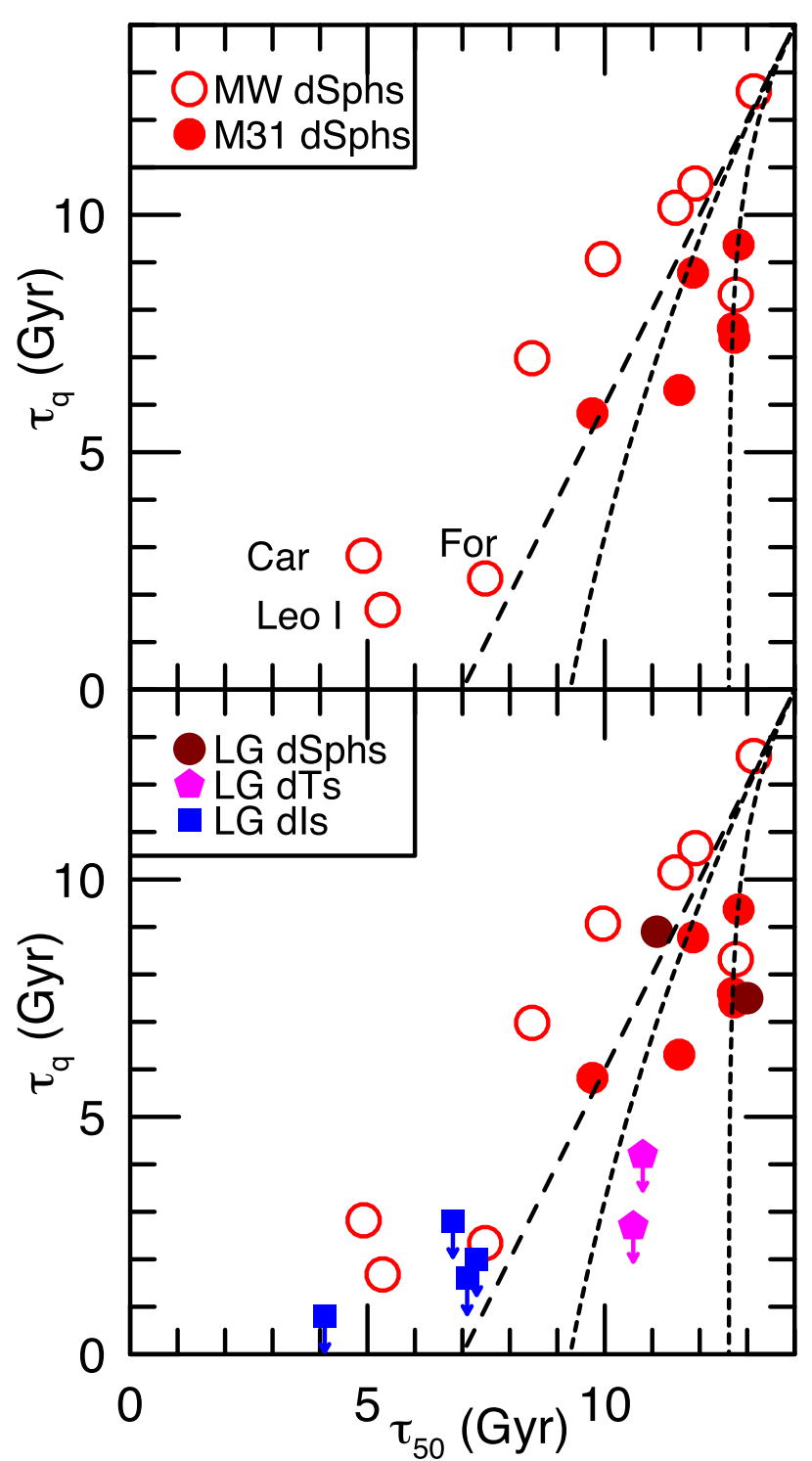

Figure 10. Upper panel: a comparison of the lookback times for $90 \%\left(\tau_{q}\right)$ and $50 \%\left(\tau_{50}\right)$ of the stars to form for the MW dSphs taken from Weisz et al. (2014a) and the ISLAndS dSphs studied here. The long dashed line represents the relationship for constant star formation ended by instantaneous quenching. The two short dashed lines are for exponentially decreasing star formation $(\tau$ models) ended by instantaneous quenching (with timescales of $2 \mathrm{Gyr}$ and 10 Gyr). The ensemble of MW satellite galaxies is roughly consistent with constant star formation with rapid quenching. The M31 satellite galaxies are roughly consistent with $\tau$ models. The M31 satellite galaxy sample shows no late-quenching galaxies as observed in the MW sample. Lower panel: we add to the top panel values of $\tau_{q}$ and $\tau_{50}$ for isolated Local Group dwarfs with oMSTO photometry. The isolated dSphs Cetus and Tucana fit in with the satellite dSphs, the dI galaxies overlap with the late-quenching MW dSphs, and the isolated dT galaxies define their own region.

SFRs after a significant delay (as seen in Figure 7). This difference could be due to the delayed effects of reionization, i.e., although neither sample is showing complete quenching by reionization, perhaps the two have been impacted differently by reionization. Alternatively, this difference could be reflecting the impact of the parent galaxies on the early evolution of the satellites. With such small samples and recognizing the complications of orbital histories, it will be difficult to draw definitive conclusions. 
Nonetheless, there appears to be evidence that the evolution of the M31 dSphs was more uniform than the evolution of the MW dSphs.

\subsection{Comparing SFH Characteristics}

In this last section, we compare the values of $\tau_{q}$ versus $\tau_{50}$. In the top panel of Figure 10, we plot $\tau_{q}$ versus $\tau_{50}$ for the MW and M31 dSph satellite samples. Here we see the very interesting trend that $\tau_{q}$ and $\tau_{50}$ are relatively well correlated for both the MW and the M31 dSphs. This is not totally unexpected since $\tau_{q}$ must always be greater than or equal to $\tau_{50}$, but galaxies that form a substantial fraction of their stars early and then quench very late (i.e., galaxies that would be in the lower right hand corner of Figure 10) are, in principle, possible, but not observed. The general trend is, to first order, consistent with relatively constant star formation ended by a quenching event. The long dashed line in Figure 10 shows $\tau_{50}=\tau_{q} / 2$, which would be the result of constant star formation followed by rapid quenching. There is good evidence for a rapid transformation of star-forming satellite dwarf galaxies into dSphs after falling into the host galaxy halo (Fillingham et al. 2015; Wetzel et al. 2015b), so the assumption of instantaneous quenching is supported. For the late-quenching galaxies, quenching by reionization is certainly not a factor, and environmental quenching associated with infall seems most likely. Of all of the possible ways to remove the gas, ram pressure stripping is the fastest (Mayer et al. 2007).

Star formation in galaxies is often modeled as exponentially decreasing ( $\tau$ models), so it is of interest to see where these models would lie in this diagnostic diagram. The short dashed lines show $\tau$ models with time constants of 2 and 10 Gyr. Since increasing the time constant will only more closely approximate constant star formation, it is clear that constant star formation is a better approximation to the SFHs of the MW dSphs. On the other hand, most of the M31 satellites can be found lying between the two $\tau$ models. Weisz et al. (2014a) found that the SFHs of dSph galaxies could be approximated by exponentially declining star formation with a $\tau$ of $5 \mathrm{Gyr}$. It appears from Figure 10 that constant star formation is a better characterization for the MW satellites, but that the M31 satellites might be better described with a range of $\tau$ models.

Gallart et al. (2015) have proposed that the dSphs can be divided into "slow" and "fast" classes based on their lifetime SFHs, and have shown that these classes do not necessarily correspond with current morphological types. In particular, some galaxies classified as dSph such as Fornax, Carina, and Leo I, are slow according to their lifetime SFHs. They propose that the distinction between a fast and a slow dwarf galaxy primarily reflects the characteristic density of the environment where they formed, with fast dwarfs assembled in higher density environments than slow dwarfs. While they do not provide a quantitative definition of fast and slow, all of the example fast galaxies have $\tau_{50} \geqslant 9$ Gyr. Figure 10 shows that all of the ISLAndS M31 dSph satellites have $\tau_{50} \geqslant$ nine Gyr, so these would all be regarded as fast while four of the nine MW dSphs would be regarded as slow. This difference between the two satellite systems can be interpreted, following Gallart et al. (2015), as indicative of a higher density environment for the formation of the M31 dSphs. This is in agreement with other indications, i.e., that M31 seems to be a more massive galaxy, with a more populous satellite system that includes more massive dwarf galaxies than the MW satellite system.
The top panel of Figure 10 reinforces the main conclusion from this section that the M31 and MW dSph populations distinguish themselves by the complete absence of latequenching dSphs in the M31 population. Of course, the ISLAndS sample is only representative and not complete, but there has been no selection bias that could have led to this result. The M31 and MW dSph samples overlap in distance from host and galaxy luminosity, so there must be some other parameter of importance. A secondary, and more subtle difference is the segregation of the M31 and MW dSph populations in this diagram. To first order, the M31 $\mathrm{dSphs}$ lie on or below the long dashed line indicating constant star formation, while the MW dSphs are all above this line. This is a re-manifestation of the trend already noted that the M31 dSphs produced half of their stars earlier than the MW $\mathrm{dSphs}$ and then were quenched at approximately the same time (discounting the late-quenching MW dSphs).

In the bottom panel of Figure 10, we have added the results from the SFHs from the six isolated Local Group dwarfs from the LCID study (from Figure 10 in Skillman et al. 2014) consisting of two dSphs (Cetus and Tucana), two dwarf transition galaxies ${ }^{19}$ (dTrans or, hereafter, dT galaxies; LGS-3 and Phoenix), and two dIs (Leo A and IC 1613). We have also added two other isolated dIs with comparable deep HST photometry: Leo T (Weisz et al. 2012) and Aquarius (Cole et al. 2014).

Note that, for the dT and dI galaxies, it is trivial to calculate the times when $90 \%$ of the present stars are in place, but then there is the concern of referring to this as a quenching time for galaxies that are not yet quenched. To recognize this ambiguity, we have plotted them at the time when $90 \%$ of the present stars had formed, but indicated these positions as upper limits. That is, if these galaxies were quenched today, this is where their points would fall in the $\tau_{q}$ versus $\tau_{50}$ diagram.

The Local Group isolated dSphs Cetus and Tucana fit in with the distribution of companion dSphs. From the point of view of SFHs, there seems to be little to distinguish the isolated dSphs from companion dSphs. Since proximity to a large galaxy appears to be a requirement for most galaxies to evolve into a dSph galaxy, the present distances of the isolated dSphs from the MW may not be representative of their entire histories. Indeed, the radial velocities of Cetus and Tucana (Lewis et al. 2007; Fraternali et al. 2009) are consistent with orbiting within the virial volume of the MW (Teyssier et al. 2012). This supports the hypothesis that there is a commonality to evolutionary paths of all of the LG dSphs in that they have all been influenced by proximity to a massive galaxy.

The nature of dT galaxies has been discussed by Skillman et al. (2003) who found that most dTs are consistent with normal dI galaxies exhibiting temporarily interrupted star formation, but that their observed density-morphology relationship indicates that environmental processes may play a role in causing their lower present day SFRs. From SFHs, Weisz et al. (2011) found the majority of the dTs to be low-mass dIs that simply lack $\mathrm{H} \alpha$ emission, but that some dTs (like Phoenix) have remarkably low gas fractions. The LCID project provided very detailed SFHs for Phoenix (Hidalgo et al. 2009) and LGS3 (Hidalgo et al. 2011) and they are included in the bottom panel of Figure 10. Interestingly, the isolated dT galaxies define

\footnotetext{
${ }^{19}$ Dwarf transition galaxies (dTrans or dT) are defined by the presence of recent star formation but the lack of very recent massive star formation (i.e., the lack of detected $\mathrm{H}$ II regions).
} 
their own region in the diagnostic diagram in Figure 10. Although there are no dSphs in the early build-up and latequenching corner of the diagram, nature is able to produce galaxies in this parameter space through the process or processes that produce dT galaxies. The dT galaxies are characterized by significant early star formation, which has decreased in amplitude in time, and thus, they occupy a region of the diagram that fits well with $\tau$ models.

Finally, the dI galaxies overlap with the late-quenching MW dSphs. ${ }^{20}$ The overlap of the dIs with the late-quenching dSphs is really quite remarkable and suggests that just a few gigayears ago the late-quenching MW dSphs would have been identical to today's dIs. This reinforces the idea of an environmental evolutionary link between the two and the conclusion that the current morphological type is not necessarily a good diagnostic of the intrinsic, long-term evolution of galaxy properties (e.g., Gallart et al. 2015). The measurement of Leo I's proper motion by Sohn et al. (2013) shows a coincidence of the quenching time with the time of the orbital perigalacticon, which suggests that Leo I may offer a prototype for the conversion of star-forming to gas-free dwarf galaxies due to processes associated with infall into the host galaxy's virial volume. Wetzel et al. (2015a) have modeled the infall histories of dwarf galaxies and identified two sources of strong environmental influence, the virial volume of the host and "group pre-processing." The challenge is now to combine SFHs and reconstructed orbits to determine whether these models provide an adequate description of the galaxies in the Local Group. Additional modeling that can reproduce satellite populations with and without late-quenching satellites will be of extreme interest.

\section{Summary and Conclusions}

We have presented HST ACS and WFC3 observations of six M31 dSph galaxies as part of the Initial Star formation and Lifetimes of Andromeda Satellites (ISLAndS) project. These six galaxies comprise a representative sample of Andromeda $\mathrm{dSph}$ satellite companion galaxies. The main goal of this program is to determine whether the SFHs of the Andromeda $\mathrm{dSph}$ satellites are significantly different from those of the MW.

Our observations reach the oMSTOs, allowing a time resolution at the oldest ages of $\sim 1 \mathrm{Gyr}$. We find that the six dSphs present a variety of SFHs, which are not strictly correlated with luminosity or present distance from M31. Specifically, we find a broad range in quenching times, but all quenching times are earlier than $\sim 6$ Gyr ago. In agreement with observations of MW companions of similar mass, there is no evidence of complete quenching of star formation by the cosmic UV background responsible for reionization, but the possibility of a degree of quenching at reionization cannot be ruled out.

We do not find significant differences between the SFHs of the three members of the vast, thin plane of satellites discovered by Ibata et al. (2013) and the three off-plane dSphs in our sample. This is further confirmation of the conclusion by Collins et al. (2015) that there is no evidence of physical differences between the in-plane and out-of-plane galaxies.

\footnotetext{
${ }^{20}$ Note that we have included Leo $\mathrm{T}$ with the $\mathrm{dI}$ galaxies with the justification that Leo $\mathrm{T}$ is simply a low-mass dI lacking $\mathrm{H} \alpha$ emission. In many regards, Leo $\mathrm{T}$ is similar to the $\mathrm{dI}$ Leo $\mathrm{P}$ with the exception that Leo $\mathrm{P}$ does have an $\mathrm{H}$ II region (McQuinn et al. 2015).
}

For a simplified comparison of SFHs and a preliminary comparison between the MW and M31 dSphs, we concentrate on the lookback times when $50 \%\left(\tau_{50}\right)$ and $90 \%\left(\tau_{q}\right)$ of the total stars are formed. A preliminary comparison of the SFHs of the ISLAndS dSphs with those MW dSph companions with similar luminosities and host distances shows that there is one primary significant difference using this diagnostic. There are no latequenching $\left(\tau_{q} \leqslant 5\right.$ Gyr) dSphs in the ISLAndS sample. Thus, modeling that can reproduce satellite populations with and without late-quenching satellites will be of extreme interest. A secondary and more subtle difference is the segregation of the M31 and MW dSph populations in a diagram of $\tau_{q}$ versus $\tau_{50}$ (i.e., our Figure 10). The M31 dSphs, on average, produced half of their stars earlier than the MW dSphs and then, on average, were quenched later (discounting the late-quenching MW dSphs). Interestingly, to first order, the ensemble of M31 dSphs are consistent with $\tau$ models, while the MW satellites are better described as having nearly constant SFRs until being rapidly quenched.

We gratefully acknowledge a very detailed and helpful report by our referee, Mario Mateo. We are also grateful for helpful advice from Aaron Dotter concerning the differences between stellar evolution libraries. Support for this work was provided by NASA through grants GO-13028 and GO-13739 from the Space Telescope Science Institute, which is operated by AURA, Inc., under NASA contract NAS5-26555. Support for D.R.W. is provided by NASA through Hubble Fellowship grant HST-HF-51331.01 awarded by the Space Telescope Science Institute. The computer network at IAC operated under the Condor software license has been used. Authors S.H. and A.A. are funded by the IAC (grant P3/94) and, with SC, by the Economy and Competitiveness Ministry of the Kingdom of Spain (grant AYA2013-42781P). Authors C.G., C.E.M.-V., and M.M. are funded by the IAC grant P/301204 and by the Spanish Ministry of Economy and Competitiveness (MINECO) under the grant (project reference AYA2014-56795-P). This research has made use of NASA's Astrophysics Data System Bibliographic Services and the NASA/IPAC Extragalactic Database (NED), which is operated by the Jet Propulsion Laboratory, California Institute of Technology, under contract with the National Aeronautics and Space Administration.

\section{Appendix \\ The Impact of Different Stellar Libraries on SFHs}

There are three main sources of uncertainties in deriving SFHs (see, e.g., discussion in Aparicio \& Hidalgo 2009): the input observational uncertainties, the statistical uncertainties of the solution, and the systematic uncertainties of the adopted isochrones from the stellar evolution libraries. In a specific test of creating synthetic photometry from one stellar evolution library and using a different stellar evolution library to derive a SFH, Aparicio \& Hidalgo (2009) showed that the systematic uncertainties associated with the adopted isochrones from the stellar evolution libraries dominated those of the numerical methodology or the observation uncertainties (when there are sufficient stars to provide reasonably small statistical uncertainties). They recommend always using more than one stellar evolution library when analyzing a real population to test for these effects. Weisz et al. (2011) and Dolphin (2012) have also shown that, for sufficiently deep photometry, the stellar evolution uncertainties, as approximated by differences 

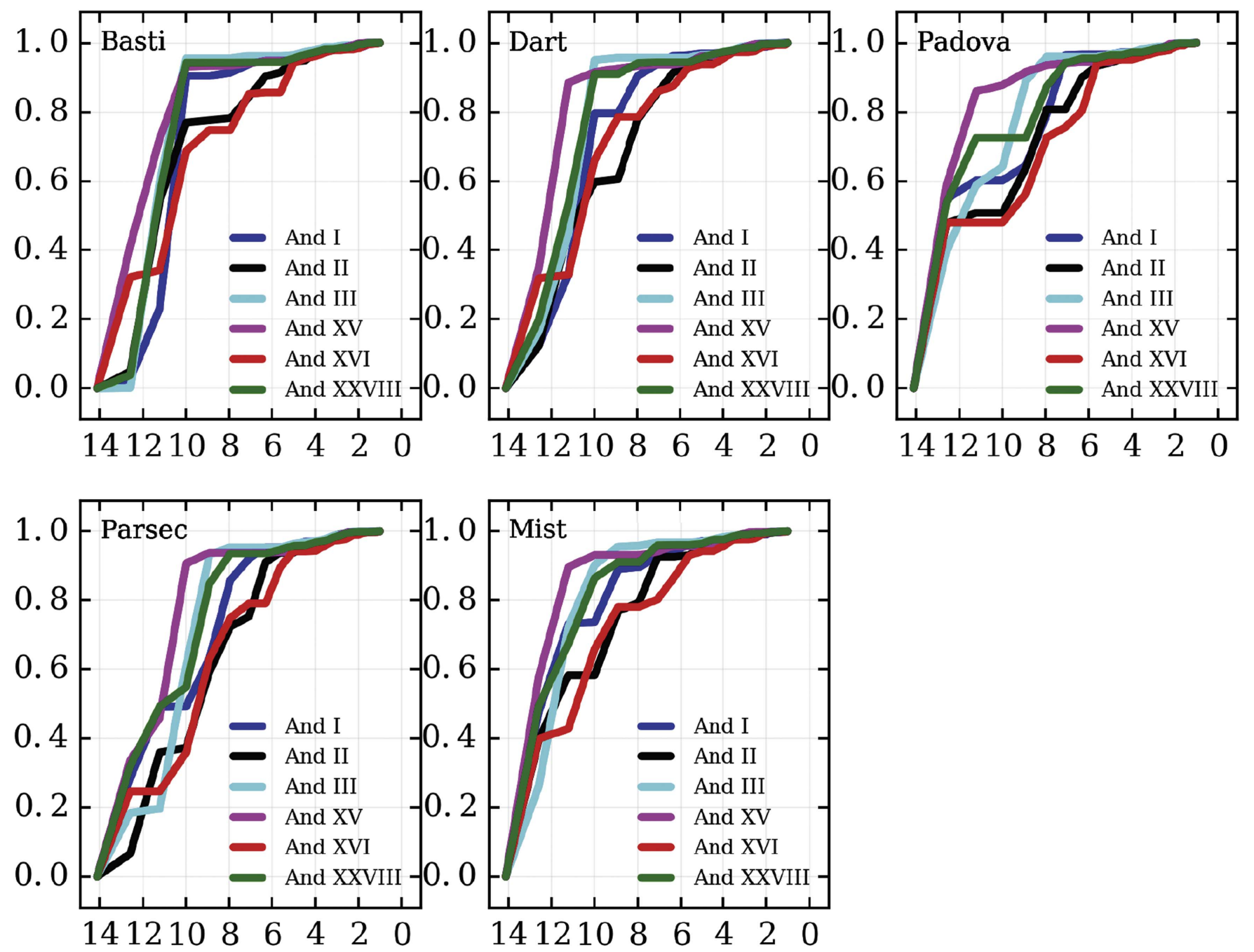

Figure 11. The cumulative SFHs for the ISLAndS galaxies as a result of using five different stellar evolution libraries (BASTI, Dartmouth, Padova, PARSEC, and MIST: Pietrinferni et al. 2004; Dotter et al. 2008; Girardi et al. 2010; Bressan et al. 2012; Choi et al. 2016). The colors are identical to those in Figure 7, and the upper right panel shows the same solutions as in Figure 7. There are systematic differences which are at least as large as the statistical uncertainties in the solutions (as can be judged from Figure 7). Note, however, that the ordering of the galaxies in their star formation properties is quite consistent from panel to panel. That is, And XV consistently shows the fastest production of its stars, while And II and And XVI are the slowest. Thus, comparisons conducted using a single stellar evolution library are likely to result in robust differences/similarities, but the absolute time frame (and details of periods of quiescence and bursts) will depend on the choice of stellar evolution library.

between stellar evolution model and their resulting libraries, represent the dominant systematic uncertainty for derived SFHs. (See also the discussion in Dotter et al. 2007, concerning the effects of varying heavy element abundance patterns.)

These differences between stellar evolution libraries can arise from differences in physical inputs and assumptions made during production. Table 1 in Gallart et al. (2005) provides a good overview of the physical ingredients and choices that are made in constructing a stellar evolution model. As examples, we list here three important parameters in stellar evolution modeling. First, the stellar libraries can change as a result of updating input data such as nuclear reaction rates. Since the determination of reaction rates of important processes continue to change (e.g., Fynbo et al. 2005) and since significant computational effort is required to produce an entire library, it is natural that differences will be found between stellar libraries with different release dates. Second, the codes can differ in how to manage the efficiency of non-canonical processes such as core overshooting. Third, different libraries make different assumptions about certain model specifications such as the adopted heavy element solar mixture, the initial He abundance at the lower metallicity (the primordial helium abundance), the assumed helium-enrichment ratio $d Y / d Z$, and the the adopted color-effective temperature relations and bolometric correction scales needed to transfer the model predictions from the theoretical plane to the various observational CMDs. Thus, differences at some level are natural and not likely to disappear. (Choi et al. 2016, their Section 7) present a very informative comparison of some of the differences between existing stellar evolution libraries.

In order to give a sense of the importance of the choice of the stellar evolution library, we present in Figure 11 a comparison of the SFHs for all of the ISLAndS sample derived using five different stellar evolution libraries (BASTI, Dartmouth, Padova, PARSEC, and MIST: Pietrinferni et al. 2004; Dotter et al. 2008; Girardi et al. 2010; Bressan et al. 2012; Choi et al. 2016). While all five plots are similar, there are systematic differences which are at least as large as the statistical 


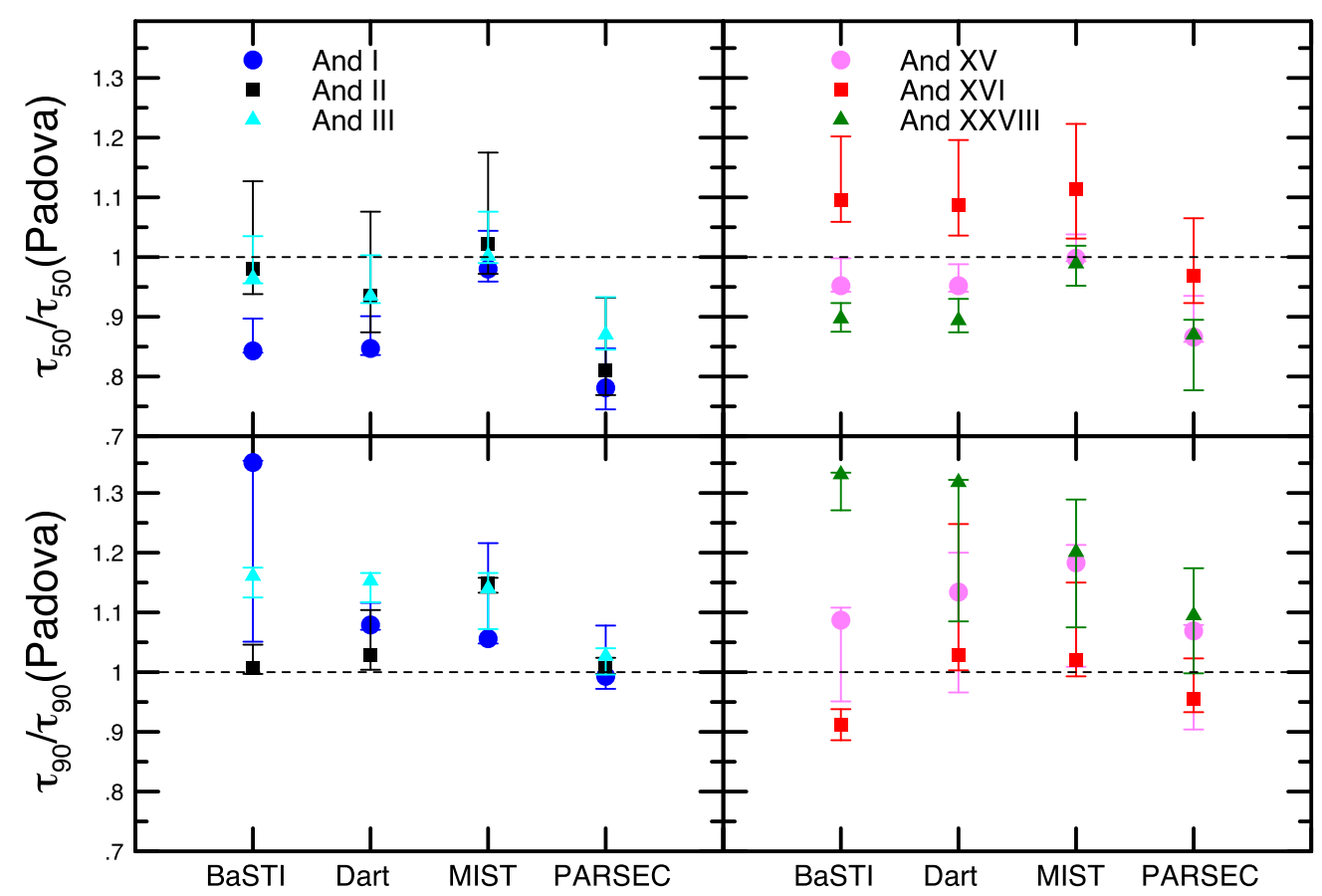

Figure 12. A comparison of the values of $\tau_{50}$ and $\tau_{q}$ for the SFHs of the ISLAndS dSphs derived by using four different stellar evolution libraries relative to the solutions using the Padova stellar evolution library. The more populous galaxies are plotted in the left panels and the less populous galaxies in the right panels. The values of $\tau_{q}$ for the more populous galaxies show general agreement (within 10\%-20\%) between the derived values (with different offsets for different libraries). Similar agreement is see for $\tau_{50}$, although the solutions for And I appear discrepant. For the less populous galaxies there is significant spread for all galaxies and all libraries, with the implication that the statistical uncertainties due to lower numbers of stars are beginning to compete with the systematic uncertainty of the choice of stellar library. When there are sufficient stars to suppress the statistical uncertainties, then the systematic uncertainty in $\tau_{q}$ (and to some degree in $\tau_{50}$ ) due to the choice of a stellar evolution library can be characterized, to first order, as a temporal offset.

uncertainties in the solutions (compare with Figure 7). Dramatic differences in the bin-to-bin SFRs are minimized in the cumulative SFHs plots, but the positions of steep changes in the SFHs can be seen to shift systematically with choice of stellar evolution library.

Note, however, that the differences are consistent between the galaxies, i.e., the MIST and Padova solutions form stars the fastest, the BASTI and Dartmouth solutions are intermediate and the PARSEC solutions form the stars the slowest. A close inspection of Figure 11 shows that there are real reasons to be optimistic. Notably, the ordering of the galaxies in their star formation properties is quite consistent from panel to panel. That is, And VX consistently shows the fastest production of its stars, while And II and And XVI are the slowest. Thus, comparisons conducted using a single stellar evolution library are likely to result in robust differences/similarities, but the absolute time frame (and details of periods of quiescence and bursts) will depend on the choice of stellar evolution library. Ideally, one can imagine that the differences between stellar evolution libraries will diminish (e.g., as nuclear reaction rates become more precisely determined and uniform between libraries).

So, what is the impact of these differences on the parameters derived in this paper, specifically $\tau_{50}$ and $\tau_{q}$ ? In Figure 12, we have plotted a comparison of the values of $\tau_{50}$ and $\tau_{q}$ for the SFHs derived from the five different stellar evolution libraries. We have divided the diagram into two parts, the more populous galaxies (And I, And II, and And III) in the left plots and the less populous galaxies (And XV, And XVI, and And XXVIII) on the right. The plot is constructed with the Padova solutions as a baseline. For the values of $\tau_{q}$ for the more populous galaxies we see that there is excellent agreement between the derived values (with offsets between the different libraries). The situation is not quite as good for $\tau_{50}$, as the solutions for And I appear discrepant. For the less populous galaxies there is significant spread, and it looks like the statistical uncertainties due to lower numbers of stars are beginning to compete with the systematic uncertainty of the choice of stellar library. The differences are of order $30 \%$ or less for all values, for all galaxies. In summary, it appears that when there are sufficient stars to suppress the statistical uncertainties, then the systematic uncertainty in $\tau_{q}$ can be characterized as a temporal offset. There is some evidence that this is also true for $\tau_{50}$, but the case is not as good as for $\tau_{q}$.

Thus, when comparing SFHs between galaxies or between groups of galaxies, it is imperative to approach the data with as uniform a methodology as possible, and especially, to use the same stellar library. Ideally, comparisons should be conducted using a variety of libraries such that a sense of the systematic uncertainty associated with the choice of the library can be achieved. While comparisons conducted using a single stellar evolution library are likely to result in robust differences/ similarities, it is primarily the absolute time frame that will depend on the choice of stellar evolution library. A goal of the ISLAndS project is to conduct uniform comparisons between MW and M31 satellite SFHs using multiple stellar evolution libraries.

\section{References}

Amorisco, N. C., Evans, N. W., \& van de Ven, G. 2014, Natur, 507, 335

Aparicio, A., Carrera, R., \& Martínez-Delgado, D. 2001, AJ, 122, 2524

Aparicio, A., \& Gallart, C. 2004, AJ, 128, 1465

Aparicio, A., \& Hidalgo, S. L. 2009, AJ, 138, 558

Aparicio, A., Hidalgo, S. L., Skillman, E., et al. 2016, ApJ, 823, 9 
Bahl, H., \& Baumgardt, H. 2014, MNRAS, 438, 2916

Beaton, R. L., Majewski, S. R., Guhathakurta, P., et al. 2007, ApJL, 658, L91 Becker, G. D., Bolton, J. S., Madau, P., et al. 2015, MNRAS, 447, 3402 Bedin, L. R., Cassisi, S., Castelli, F., et al. 2005, MNRAS, 357, 1038 Bell, E. F., Slater, C. T., \& Martin, N. F. 2011, ApJL, 742, L15 Bellazzini, M. 2008, MmSAI, 79, 440

Benítez-Llambay, A., Navarro, J. F., Abadi, M. G., et al. 2013, ApJL, 763, L41 Benítez-Llambay, A., Navarro, J. F., Abadi, M. G., et al. 2016, MNRAS, 456, 1185

Benson, A. J., Bower, R. G., Frenk, C. S., et al. 2003, ApJ, 599, 38

Benson, A. J., Frenk, C. S., Lacey, C. G., Baugh, C. M., \& Cole, S. 2002, MNRAS, 333, 177

Bernard, E. J., Ferguson, A. M. N., Barker, M. K., et al. 2012, MNRAS, 420,2625

Bernard, E. J., Ferguson, A. M. N., Chapman, S. C., et al. 2015a, MNRAS, 453, L113

Bernard, E. J., Ferguson, A. M. N., Richardson, J. C., et al. 2015b, MNRAS, 446, 2789

Bernard, E. J., Monelli, M., Gallart, C., et al. 2009, ApJ, 699, 1742

Bernard, E. J., Monelli, M., Gallart, C., et al. 2010, ApJ, 712, 1259

Bono, G., Stetson, P. B., Walker, A. R., et al. 2010, PASP, 122, 651

Bovill, M. S., \& Ricotti, M. 2009, ApJ, 693, 1859

Bovill, M. S., \& Ricotti, M. 2011, ApJ, 741, 18

Boylan-Kolchin, M., Bullock, J. S., \& Kaplinghat, M. 2011, MNRAS, 415, L40

Boylan-Kolchin, M., Bullock, J. S., \& Kaplinghat, M. 2012, MNRAS, 422, 1203

Boylan-Kolchin, M., Weisz, D. R., Bullock, J. S., \& Cooper, M. C. 2016, MNRAS, 462, L51

Boylan-Kolchin, M., Weisz, D. R., Johnson, B. D., et al. 2015, MNRAS, 453, 1503

Brasseur, C. M., Martin, N. F., Macciò, A. V., Rix, H.-W., \& Kang, X. 2011, ApJ, 743, 179

Bressan, A., Marigo, P., Girardi, L., et al. 2012, MNRAS, 427, 127

Brown, T. M., Smith, E., Ferguson, H. C., et al. 2006, ApJ, 652, 323

Brown, T. M., Tumlinson, J., Geha, M., et al. 2014, ApJ, 796, 91

Buck, T., Macciò, A. V., \& Dutton, A. A. 2015, ApJ, 809, 49

Bullock, J. S. 2010, arXiv:1009.4505

Bullock, J. S., \& Johnston, K. V. 2005, ApJ, 635, 931

Bullock, J. S., Kravtsov, A. V., \& Weinberg, D. H. 2000, ApJ, 539, 517

Busha, M. T., Alvarez, M. A., Wechsler, R. H., Abel, T., \& Strigari, L. E. 2010, ApJ, 710, 408

Carrera, R., Aparicio, A., Martínez-Delgado, D., \& Alonso-García, J. 2002, AJ, 123,3199

Carretta, E., Gratton, R. G., Clementini, G., \& Fusi Pecci, F. 2000, ApJ, 533,215

Choi, J., Dotter, A., Conroy, C., et al. 2016, ApJ, 823, 102

Cole, A. A., Skillman, E. D., Tolstoy, E., et al. 2007, ApJL, 659, L17

Cole, A. A., Weisz, D. R., Dolphin, A. E., et al. 2014, ApJ, 795, 54

Collins, M. L. M., Chapman, S. C., Rich, R. M., et al. 2013, ApJ, 768, 172

Collins, M. L. M., Chapman, S. C., Rich, R. M., et al. 2014, ApJ, 783, 7

Collins, M. L. M., Martin, N. F., Rich, R. M., et al. 2015, ApJL, 799, L13

Conn, A. R., Ibata, R. A., Lewis, G. F., et al. 2012, ApJ, 758, 11

Conn, A. R., Lewis, G. F., Ibata, R. A., et al. 2011, ApJ, 740, 69

Crnojević, D., Ferguson, A. M. N., Irwin, M. J., et al. 2014, MNRAS, 445,3862

da Costa, G. S., Armandroff, T. E., \& Caldwell, N. 2002, AJ, 124, 332

da Costa, G. S., Armandroff, T. E., Caldwell, N., \& Seitzer, P. 1996, AJ, 112,2576

da Costa, G. S., Armandroff, T. E., Caldwell, N., \& Seitzer, P. 2000, AJ, 119,705

Deason, A. J., Belokurov, V., Evans, N. W., \& Johnston, K. V. 2013, ApJ, 763,113

de Boer, T. J. L., Tolstoy, E., Hill, V., et al. 2012a, A\&A, 539, A103

de Boer, T. J. L., Tolstoy, E., Hill, V., et al. 2012b, A\&A, 544, A73

de Boer, T. J. L., Tolstoy, E., Lemasle, B., et al. 2014, A\&A, 572, A10

Dekel, A., \& Silk, J. 1986, ApJ, 303, 39

Dijkstra, M., Haiman, Z., Rees, M. J., \& Weinberg, D. H. 2004, ApJ, 601, 666

Dolphin, A. E. 2000, PASP, 112, 1383

Dolphin, A. E. 2002, MNRAS, 332, 91

Dolphin, A. E. 2012, ApJ, 751, 60

Dolphin, A. E. 2013, ApJ, 775, 76

Dolphin, A. E. 2016, ApJ, 825, 153

Dotter, A. 2016, ApJS, 222, 8

Dotter, A., Chaboyer, B., Jevremović, D., et al. 2007, AJ, 134, 376

Dotter, A., Chaboyer, B., Jevremović, D., et al. 2008, ApJS, 178, 89
Duane, S., Kennedy, A. D., Pendleton, B. J., \& Roweth, D. 1987, PhLB, 195,216

Efstathiou, G. 1992, MNRAS, 256, 43P

Fan, X., Strauss, M. A., Becker, R. H., et al. 2006, AJ, 132, 117

Fardal, M. A., Weinberg, M. D., Babul, A., et al. 2013, MNRAS, 434, 2779

Ferguson, A. M. N., Irwin, M. J., Ibata, R. A., Lewis, G. F., \& Tanvir, N. R. 2002, AJ, 124, 1452

Ferraro, F. R., Sabbi, E., Gratton, R., et al. 2006, ApJL, 647, L53

Fillingham, S. P., Cooper, M. C., Wheeler, C., et al. 2015, MNRAS, 454, 2039

Fraternali, F., Tolstoy, E., Irwin, M. J., \& Cole, A. A. 2009, A\&A, 499, 121

Fynbo, H. O. U., Diget, C. A., Bergmann, U. C., et al. 2005, Natur, 433, 136

Gallart, C., Monelli, M., Mayer, L., et al. 2015, ApJL, 811, L18

Gallart, C., Zoccali, M., \& Aparicio, A. 2005, ARA\&A, 43, 387

Garrison-Kimmel, S., Boylan-Kolchin, M., Bullock, J. S., \& Kirby, E. N. 2014, MNRAS, 444, 222

Geha, M., Weisz, D., Grocholski, A., et al. 2015, ApJ, 811, 114

Gillet, N., Ocvirk, P., Aubert, D., et al. 2015, ApJ, 800, 34

Girardi, L., Williams, B. F., Gilbert, K. M., et al. 2010, ApJ, 724, 1030

Gnedin, N. Y., \& Kravtsov, A. V. 2006, ApJ, 645, 1054

Gosnell, N. M., Mathieu, R. D., Geller, A. M., et al. 2014, ApJL, 783, L8

Gosnell, N. M., Mathieu, R. D., Geller, A. M., et al. 2015, ApJ, 814, 163

Governato, F., Willman, B., Mayer, L., et al. 2007, MNRAS, 374, 1479

Graus, A. S., Bullock, J. S., Boylan-Kolchin, M., \& Weisz, D. R. 2016, MNRAS, 456, 477

Grcevich, J., \& Putman, M. E. 2009, ApJ, 696, 385

Grebel, E. K., \& Gallagher, J. S., III 2004, ApJL, 610, L89

Grillmair, C. J., Mould, J. R., Holtzman, J. A., et al. 1998, AJ, 115, 144

Hammer, F., Yang, Y., Fouquet, S., et al. 2013, MNRAS, 431, 3543

Hidalgo, S. L., Aparicio, A., Martínez-Delgado, D., \& Gallart, C. 2009, ApJ, 705,704

Hidalgo, S. L., Aparicio, A., Skillman, E., et al. 2011, ApJ, 730, 14

Hidalgo, S. L., Monelli, M., Aparicio, A., et al. 2013, ApJ, 778, 103

Hill, J. R., Ferrarese, L., Stetson, P. B., et al. 1998, ApJ, 496, 648

Ho, N., Geha, M., Munoz, R. R., et al. 2012, ApJ, 758, 124

Ho, N., Geha, M., Tollerud, E. J., et al. 2015, ApJ, 798, 77

Holtzman, J. A., Afonso, C., \& Dolphin, A. 2006, ApJS, 166, 534

Hurley-Keller, D., Mateo, M., \& Nemec, J. 1998, AJ, 115, 1840

Huxor, A. P., Ferguson, A. M. N., Tanvir, N. R., et al. 2011, MNRAS, 414, 770

Ibata, R., Chapman, S., Ferguson, A. M. N., et al. 2004, MNRAS, 351, 117

Ibata, R., Irwin, M., Lewis, G., Ferguson, A. M. N., \& Tanvir, N. 2001, Natur, 412, 49

Ibata, R. A., Ibata, N. G., Lewis, G. F., et al. 2014, ApJL, 784, L6

Ibata, R. A., Lewis, G. F., Conn, A. R., et al. 2013, Natur, 493, 62

Kalirai, J. S., Beaton, R. L., Geha, M. C., et al. 2010, ApJ, 711, 671

Kauffmann, G., White, S. D. M., \& Guiderdoni, B. 1993, MNRAS, 264, 201

Klypin, A., Kravtsov, A. V., Valenzuela, O., \& Prada, F. 1999, ApJ, 522, 82

Kormendy, J. 1985, ApJ, 295, 73

Kormendy, J., \& Bender, R. 2012, ApJS, 198, 2

Kravtsov, A. V., Gnedin, O. Y., \& Klypin, A. A. 2004, ApJ, 609, 482

Lewis, G. F., Ibata, R. A., Chapman, S. C., et al. 2007, MNRAS, 375, 1364

Libeskind, N. I., Hoffman, Y., Tully, R. B., et al. 2015, MNRAS, 452, 1052

Makarov, D., Makarova, L., Rizzi, L., et al. 2006, AJ, 132, 2729

Mapelli, M., Ripamonti, E., Battaglia, G., et al. 2009, MNRAS, 396, 1771

Mapelli, M., Ripamonti, E., Tolstoy, E., et al. 2007, MNRAS, 380, 1127

Marconi, M., Coppola, G., Bono, G., et al. 2015, ApJ, 808, 50

Martin, N. F., Ibata, R. A., Lewis, G. F., et al. 2016, ApJ, 833, 167

Martin, N. F., Schlafly, E. F., Slater, C. T., et al. 2013a, ApJL, 779, L10

Martin, N. F., Slater, C. T., Schlafly, E. F., et al. 2013b, ApJ, 772, 15

Mateo, M., Harris, H. C., Nemec, J., \& Olszewski, E. W. 1990, AJ, 100 469

Mateo, M. L. 1998, ARA\&A, 36, 435

Mayer, L., Governato, F., Colpi, M., et al. 2001a, ApJL, 547, L123

Mayer, L., Governato, F., Colpi, M., et al. 2001b, ApJ, 559, 754

Mayer, L., Kazantzidis, S., Mastropietro, C., \& Wadsley, J. 2007, Natur, 445, 738

Mayer, L., Mastropietro, C., Wadsley, J., Stadel, J., \& Moore, B. 2006, MNRAS, 369, 1021

McConnachie, A. W. 2012, AJ, 144, 4

McConnachie, A. W., Arimoto, N., \& Irwin, M. 2007, MNRAS, 379, 379

McConnachie, A. W., \& Côté, P. 2010, ApJL, 722, L209

McConnachie, A. W., Huxor, A., Martin, N. F., et al. 2008, ApJ, 688, 1009

McConnachie, A. W., \& Irwin, M. J. 2006a, MNRAS, 365, 902

McConnachie, A. W., \& Irwin, M. J. 2006b, MNRAS, 365, 1263

McConnachie, A. W., Irwin, M. J., Ferguson, A. M. N., et al. 2004, MNRAS, 350,243 
McConnachie, A. W., Irwin, M. J., Ferguson, A. M. N., et al. 2005, MNRAS, 356, 979

McConnachie, A. W., Irwin, M. J., Ibata, R. A., et al. 2003, MNRAS, 343, 1335

McQuinn, K. B. W., Skillman, E. D., Berg, D., et al. 2013, AJ, 146, 145

McQuinn, K. B. W., Skillman, E. D., Dolphin, A., et al. 2015, ApJ, 812, 158

Meylan, G., \& Heggie, D. C. 1997, A\&ARv, 8, 1

Mighell, K. J., \& Burke, C. J. 1999, AJ, 118, 366

Momany, Y., Held, E. V., Saviane, I., et al. 2007, A\&A, 468, 973

Monachesi, A., Trager, S. C., Lauer, T. R., et al. 2012, ApJ, 745, 97

Monelli, M., Cassisi, S., Mapelli, M., et al. 2012, ApJ, 744, 157

Monelli, M., Martínez-Vázquez, C. E., Bernard, E. J., et al. 2016, ApJ, 819,147

Monelli, M., Pulone, L., Corsi, C. E., et al. 2003, AJ, 126, 218

Monelli, M., Hidalgo, S. L., Stetson, P. B., et al. 2010a, ApJ, 720, 1225

Monelli, M., Gallart, C., Hidalgo, S. L., et al. 2010b, ApJ, 722, 1864

Moore, B. 1999, ApJ, 524, 19

Ocvirk, P., \& Aubert, D. 2011, MNRAS, 417, L93

Ocvirk, P., Gillet, N., Aubert, D., et al. 2014, ApJ, 794, 20

Okamoto, S., Arimoto, N., Yamada, Y., \& Onodera, M. 2008, A\&A, 487, 103

Okamoto, S., Arimoto, N., Yamada, Y., \& Onodera, M. 2012, ApJ, 744, 96

Okamoto, T., Gao, L., \& Theuns, T. 2008, MNRAS, 390, 920

Olszewski, E. W., \& Aaronson, M. 1985, AJ, 90, 2221

Oñorbe, J., Boylan-Kolchin, M., Bullock, J. S., et al. 2015, MNRAS, 454, 2092

Patej, A., \& Loeb, A. 2015, ApJL, 815, L28

Pawlowski, M. S., Famaey, B., Jerjen, H., et al. 2014, MNRAS, 442, 2362

Phillips, J. I., Cooper, M. C., Bullock, J. S., \& Boylan-Kolchin, M. 2015, MNRAS, 453, 3839

Pietrinferni, A., Cassisi, S., Salaris, M., \& Castelli, F. 2004, ApJ, 612, 168

Planck Collaboration, Ade, P. A. R., Aghanim, N., et al. 2016, A\&A, 594, A13

Richardson, J. C., Irwin, M. J., McConnachie, A. W., et al. 2011, ApJ, 732, 76

Ricotti, M., \& Gnedin, N. Y. 2005, ApJ, 629, 259

Rizzi, L., Tully, R. B., Makarov, D., et al. 2007, ApJ, 661, 815

Sadoun, R., Mohayaee, R., \& Colin, J. 2014, MNRAS, 442, 160

Sandage, A. R. 1953, AJ, 58, 61

Santana, F. A., Muñoz, R. R., Geha, M., et al. 2013, ApJ, 774, 106

Sawala, T., Frenk, C. S., Fattahi, A., et al. 2016, MNRAS, 457, 1931

Sawala, T., Scannapieco, C., Maio, U., \& White, S. 2010, MNRAS, 402, 1599

Schlafly, E. F., \& Finkbeiner, D. P. 2011, ApJ, 737, 103
Schlegel, D. J., Finkbeiner, D. P., \& Davis, M. 1998, ApJ, 500, 525

Sirianni, M., Jee, M. J., Benítez, N., et al. 2005, PASP, 117, 1049

Skillman, E. D., Côté, S., \& Miller, B. W. 2003, AJ, 125, 593

Skillman, E. D., Hidalgo, S. L., Weisz, D. R., et al. 2014, ApJ, 786, 44

Slater, C. T., Bell, E. F., \& Martin, N. F. 2011, ApJL, 742, L14

Slater, C. T., Bell, E. F., Martin, N. F., Tollerud, E. J., \& Ho, N. 2015, ApJ, 806,230

Sohn, S. T., Besla, G., van der Marel, R. P., et al. 2013, ApJ, 768, 139

Somerville, R. S. 2002, ApJL, 572, L23

Songaila, A. 2004, AJ, 127, 2598

Stetson, P. B. 1994, PASP, 106, 250

Susa, H., \& Umemura, M. 2004, ApJ, 600, 1

Tassis, K., Abel, T., Bryan, G. L., \& Norman, M. L. 2003, ApJ, 587, 13

Teyssier, M., Johnston, K. V., \& Kuhlen, M. 2012, MNRAS, 426, 1808

Tollerud, E. J., Beaton, R. L., Geha, M. C., et al. 2012, ApJ, 752, 45

Tollerud, E. J., Boylan-Kolchin, M., \& Bullock, J. S. 2014, MNRAS, 440,3511

Tollerud, E. J., Geha, M. C., Vargas, L. C., \& Bullock, J. S. 2013, ApJ, 768, 50

van den Bergh, S. 1994a, AJ, 107, 1328

van den Bergh, S. 1994b, ApJ, 428, 617

van den Bergh, S. 1999, A\&ARv, 9, 273

Vargas, L. C., Geha, M. C., \& Tollerud, E. J. 2014, ApJ, 790, 73

Walker, M. G., Mateo, M., Olszewski, E. W., et al. 2009, ApJ, 704, 1274

Watkins, L. L., Evans, N. W., \& An, J. H. 2010, MNRAS, 406, 264

Weinmann, S. M., Macciò, A. V., Iliev, I. T., Mellema, G., \& Moore, B. 2007, MNRAS, 381, 367

Weisz, D. R., Dalcanton, J. J., Williams, B. F., et al. 2011, ApJ, 739, 5

Weisz, D. R., Dolphin, A. E., Skillman, E. D., et al. 2014a, ApJ, 789, 147

Weisz, D. R., Dolphin, A. E., Skillman, E. D., et al. 2014b, ApJ, 789, 148

Weisz, D. R., Johnson, B. D., \& Conroy, C. 2014c, ApJL, 794, L3

Weisz, D. R., Skillman, E. D., Hidalgo, S. L., et al. 2014d, ApJ, 789, 24

Weisz, D. R., Zucker, D. B., Dolphin, A. E., et al. 2012, ApJ, 748, 88

Wetzel, A. R., Deason, A. J., \& Garrison-Kimmel, S. 2015a, ApJ, 807, 49

Wetzel, A. R., Hopkins, P. F., Kim, J.-h., et al. 2016, ApJL, 827, L23

Wetzel, A. R., Tollerud, E. J., \& Weisz, D. R. 2015b, ApJL, 808, L27

Wheeler, C., Oñorbe, J., Bullock, J. S., et al. 2015, MNRAS, 453, 1305

Williams, B. F., Dalcanton, J. J., Dolphin, A. E., et al. 2015, ApJ, 806,48

Wolf, J., Martinez, G. D., Bullock, J. S., et al. 2010, MNRAS, 406, 1220 